\title{
Towards a content-based epistemic measure in physics
}

\author{
Amogh Sirnoorkar ${ }^{*}$ \\ Department of Physics, Gurukul Pre-University Independent College of Science, \\ Kalaburagi 585102, India
}

Anwesh Mazumdar ${ }^{\dagger}$ and Arvind Kumar

Homi Bhabha Centre for Science Education (TIFR), V. N. Purav Marg, Mumbai 400088, India

(Received 8 February 2019; revised manuscript received 8 August 2019; published 16 January 2020)

\begin{abstract}
We elaborate on a new approach of assessing content-based epistemic clarity of college physics students in terms of their ability to discriminate between different epistemic warrants for propositions in a chained argument in physics. A threefold classification (nominal, physical, and mathematical) of warrants is used, with each class split into a number of distinct epistemic categories. The assessment tool consists of a questionnaire, illustrated here for a standard undergraduate physics derivation, in which the student chooses the appropriate epistemic category (from an appended list) for each proposition in the given derivation. The tool includes a brief supplementary orientation note for clarifying the meaning of different categories. It is tested for reliability and internal consistency. Correlation of content knowledge, ascertained independently, with epistemic clarity is investigated. Surveys on large and diverse samples of physics undergraduates carried out with or without the orientation note reveal a variety of epistemic errors, some common among all groups of students, and some that are good discriminators. The epistemic measure obtained from such a tool is, however, limited in scope by its narrow definition. The construct validity of the tool and the pedagogic relevance of the work are discussed.
\end{abstract}

DOI: 10.1103/PhysRevPhysEducRes.16.010103

\section{INTRODUCTION}

The role of epistemic beliefs in students' understanding of physics has been a subject of continuing attention in physics education research [1-5]. Several well-known instruments for assessing beliefs about physics and attitudes to learning physics such as MPEX [6], CLASS [7] exist in the literature. These surveys usually do not involve any explicit physics content. Consequently, there seems to be a clear separation between tools that assess students' conceptual knowledge (the concept inventories, for example) and those that assess beliefs and attitudes, though the latter often examine correlations between epistemic beliefs and conceptual understanding.

In an earlier work, we have suggested that students' epistemic understanding can be assessed in the context of how they look at standard mathematical derivations in physics [8]. This approach no doubt greatly limits the

\footnotetext{
*samogh@phys.ksu.edu

anwesh@tifr.res.in

arvind.kumar1943@gmail.com
}

Published by the American Physical Society under the terms of the Creative Commons Attribution 4.0 International license. Further distribution of this work must maintain attribution to the author(s) and the published article's title, journal citation, and DOI. meaning and scope of the term "epistemic understanding" but has the advantage that a tool can be constructed that gives a reliable and quantitative measure of students' epistemic understanding in the narrow sense defined here. It also has pedagogic relevance. This paper is basically a more systematic exposition of the same approach using a more structured tool over a bigger and diverse sample of students.

The theoretical framework for developing our new measure draws on argumentation research $[9,10]$. Where does epistemics enter in an argument or a chain of arguments involved in a derivation in physics? It is in the warrant or justification of the argument. Unlike mathematics, the source of knowledge that warrants an argument in physics is not just logic phrased qualitatively or mathematically. The source very often is empirical knowledge. Sometimes, physical reasoning can be heuristic, with no empirical or logical necessity (see Sec. II A). Also, mathematical reasoning in physics is not necessarily algebraic. Sometimes diagram-based or geometric reasoning is criti$\mathrm{cal}$, and a physical argument may necessitate visuospatial representation. Lastly, physics reasoning involves symbolization (choosing a symbol for a physical quantity), or verbalization (giving name to a certain property, etc.), or adopting a convention widely accepted in the physics community. Since these are inherently arbitrary, they have no logical or empirical warrant. The tool that we are 
looking for aims to measure students' ability to identify the warrants in the sequence of propositions that build up a physical argument.

There can be tricky issues surrounding these warrants. Are laws of physics at the most basic level mere definitions? Is some choice of physical concepts conventional? If empirical knowledge is theory laden, can we regard it as an epistemic category distinct from that of theoretical concepts? And so on. Our aim is not to get involved in these philosophical subtleties, but rather to develop a simple practical tool to measure the construct "epistemic ability" in the sense defined above. However, there are significant issues about the construct validity of the tool, which we examine in the concluding section. We argue that despite this the tool has much relevance in physics education.

\section{METHODOLOGY}

The methodology involved choosing a suitable mathematical derivation of some standard result or theorem in undergraduate physics and developing a questionnaire around it that aims to test students' ability to discriminate between different epistemic categories for the propositions appearing in the derivation. This diagnostic tool was then administered on student samples drawn from different colleges in the country and the responses analyzed.

\section{A. The epistemic warrants}

The earlier work [8] was based on a set of epistemic categories underlying the propositions involved in a particular thermodynamic derivation. However, when we attempted to expand the scope across different areas of undergraduate physics, it seemed clear that the set of epistemic warrants needed to be expanded and refined. After scrutinizing a number of derivations, a threefold classification suggested itself: mathematical or logical, physical and nominal. As remarked before, this classification can open up much philosophical debate. However, we define them by rather simple pragmatic criteria. A mathematical warrant is something that any mathematically trained person can offer with little knowledge of the physics context. It could be just a mathematical result found in mathematics texts on algebra, geometry, or calculus, etc. Or it could be a mathematical or logical deduction from previous propositions of any type. A pure logical warrant does not even need any mathematics. We combine them into one category since neither needs any physical inputs.

The physical warrant means some physics is needed as an input to the proposition. In this work we have much improved and fine structured this category. In addition to the usual physical inputs like experimental facts, principles and laws, approximation and simplification, etc., some other important reasoning types common in physics have been added.

A significant new category of physical reasoning introduced here is diagram-based reasoning. Strictly, diagram-based reasoning can perhaps always be replaced by algebraic reasoning combined with physical inputs, but in practice this would be often cumbersome. For example, in geometrical optics, given the well-known lens formulas, the position and magnification of the image follow algebraically from them, but its real or virtual nature would be hard to determine without the use of reasoning based on ray diagrams. In the same way, physical inferences on multiphase equilibria would be hard to arrive at without reasoning based on phase diagrams in thermodynamics. Other examples of this category are the H-R diagram in astrophysics and the Feynman diagrams in quantum field theory. On the other hand, merely drawing a diagram for reference or facilitation without employing any physical reasoning based on it is not diagram-based reasoning though it may be useful for learning and instruction. Contemporary scholarship in cognitive science seems to emphasize visuospatial and diagrammatic reasoning $[11,12]$, and we believe it should be viewed as a characteristically different type of physical reasoning.

Next, physical reasoning often entails a convenient choice of system, axes or frame of reference, which implies no loss of generality. Again this may not have a strict logical necessity, but is part of pragmatic practice in physics to handle a physical situation. Lastly, there is much use of heuristics in physics, and a student should be able to see if an argument is a deduction from the preceding propositions or involves heuristic reasoning. [For example, from the similarity of the entropy formula of black body radiation (in Wien's limit) with that of a molecular gas, Einstein conjectured the corpuscular picture of radiation, a classic example of heuristic reasoning.] Of course, a heuristic step, a conjectured generalization, could well be a deduction in a more rigorous derivation or a more basic theory; thus the epistemic category that applies to a proposition is to be ascertained in the framework of the given derivation.

A nominal proposition is one that requires no physical or mathematical warrant. We identify three different categories in the nominal class: symbolization, verbalization, and convention. Symbolization usually involves choosing alphabetical letters to represent physical quantities, e.g., $\sigma$ for electrical conductivity, $h$ for Planck's constant, etc. Verbalization is giving names to some properties and processes (e.g., isochoric for constant volume process, "charm" to denote a type of quark, etc.). The names chosen may or may not be suggestive of the intended meaning in physics. Convention as defined here is neither symbolization nor verbalization, but like them is still an arbitrary choice often, if not always, shared by the physics community (e.g., taking direction of electric current, by convention, to be opposite to that of electron motion in a wire in a circuit, or choosing the right side of the real number line to denote positive numbers, etc.). The essence of the nominal class as a whole is that a different choice of a 
symbol, a name, or a convention has no bearing on the substance or even convenience of the treatment of a physical problem.

The nominal propositions involving arbitrary choice of symbols, names, and conventions are epistemically different from choices made in physics aimed to facilitate handling of physical situations - the latter belong to the class of physical propositions as indicated before. For example, aligning the $z$ axis along the axis of symmetry in a problem is significantly convenient for its treatment without involving any loss of generality. This is a physical, not a nominal proposition. On the other hand, whether to take thermodynamic work to mean work done by the system or on the system has no bearing on the convenience or substance in terms of the physics of the problem. It is a nominal convention.

Based on the threefold classification (mathematical, physical, and nominal) and their subcategorization along the lines described above, we arrived at 11 distinct epistemic categories characterizing propositions in physics.

We must note at the outset that since our description of each epistemic category appearing on each page of the questionnaire is very brief, its precise intended meaning as distinct from other categories may on occasions be ambiguous, and this could pose problems in data interpretation. To deal with this issue we have carried out independent surveys on two versions of the tool: Tool 1, in which the categories were simply listed briefly, and Tool 2, in which we supplemented the same tool with a one-page orientation note that clarified these categories, many with examples (see Figs. 14-18 in the Appendix). Unsurprisingly, Tool 2 turned out to have better test statistics. Accordingly, a detailed analysis of epistemic errors is presented for data obtained from Tool 2 only (see Sec. III C). Some information on Tool 1 data is, however, included for discussion at the end.

\section{B. The tool}

The questionnaire evidently had to be based on a specific derivation in undergraduate physics. As in our earlier work, we kept quantum mechanics and relativity out of the tool, since the epistemic issues in these areas are complex and even contentious. We looked for a derivation that had diversity of epistemic categories, and, in particular, involved some diagrammatic reasoning absent in our earlier work. After looking through several possible candidates for the purpose, we finally arrived at the derivation of the well-known Bernoulli's theorem in fluid mechanics as the topic for our questionnaire.

The proof of the theorem was broken down in 36 simple propositions. The list of 11 distinct epistemic categories mentioned earlier was appended to the proof. This constituted the first version of the tool (Tool 1) that was administered to a large sample of undergraduate students (Survey 1, see Sec. II D). In the second version of the tool
(Tool 2), administered to a different sample (Survey 2 in Sec. II D), an orientation note was added aimed at disambiguation of the meaning of each category. No change was made in the questionnaire itself. In each version, the student was asked to choose the epistemic category appropriate to each proposition. The number of correct responses determined the total epistemic score $\left(S_{E}\right)$ of the student. This score is always expressed in percentage henceforth.

An important aspect of the work is to see the connection between students' content knowledge and epistemic clarity. By content knowledge we mean knowledge of a wide range of topics in physics expected at the academic stage of students. It was not feasible to incorporate a separate content-based test along with the epistemic questionnaire in the tool. We therefore decided to seek their marks (referred to as "academic marks" in the rest of the paper to distinguish from epistemic scores) in physics and mathematics at the Higher Secondary Board examination (high school in the U.S.). Although using academic marks obtained a year or two earlier as an indicator of content knowledge may be perceived as a limitation, this was still better than using their marks in the latest college examinations since the latter would differ in standards widely. Moreover, it is well known in the country that students take the Higher Secondary examination seriously, since it is widely perceived to be vital for their careers, in particular, for entry to colleges or other highly coveted institutions of higher education in science and engineering in the country.

The level and scope of Higher Secondary syllabus in India is roughly intermediate between that of high school and the first two years of college in the U.S. Syllabi, in general, tend to be content intensive in the country at all levels. For example, physics books only slightly lower in coverage than the book by Resnick and Halliday are widely used at this level. (Of course, how much of all this is truly absorbed by the average student is a separate issue.) In particular, elementary fluid dynamics, including Bernoulli's theorem, is part of the syllabus. However, taking into account the maturity desirable for answering the questionnaire of our tool, it seems appropriate to administer the tool to second or third year undergraduate students (roughly equivalent to upper division level in the U.S.). Accordingly, Survey 1 had only a small (7\%) component of first year students, while Survey 2 did not include them at all. Of course, the tool (or a variant of it with a different derivation) can be administered to even graduate students.

\section{Pilot testing and expert validation}

Tool 1 was first administered to three post-graduate physics students with no orientation prior or clarification after the test. They were subsequently interviewed separately. This led to some rewording of the propositions and epistemic categories. The content validation of the tool was then carried out by matching responses to the questionnaire 
by experts. This included the three authors and two external experts who were well versed in the subject, but had no exposure to the tool or the epistemic categories, at all. A few disagreements were debated and sorted out which again resulted in some rewording and minor modification of the questionnaire. The questionnaire was then finalized through consensus.

The revised Tool 2 carrying the orientation note with the same questionnaire was sent to two external experts, different from the earlier experts, again without any prior exposure to the tool or the epistemic categories. One of the experts sent responses in total agreement with ours. The other expert initially differed with the authors in regarding S7, S8, and S19 (dealing with notations such as $\Delta t, \Delta V, \Delta K)$ as belonging to $\mathrm{P} 1$ but was convinced after discussion that they all belonged to N1 (students, too, did not assign these to P1, as we find later). However, he insisted on assigning proposition S35 to M2, in disagreement with our choice P6. We continue to regard our choice P6 more appropriate; see the clarification under the category P6 in Sec. III C 8.

It is clear that even with the orientation note, the tool does not have perfect expert validation. It is unlikely that a test of this nature will ever have total expert agreement. This fact, however, does not, in our view, detract from the educational use of the tool. See Sec. IV for further remarks on this issue.

\section{Sample}

The sample for Survey 1 (using Tool 1) consisted of 504 students (first, second, and third year undergraduates) from 12 different colleges or institutes at 5 locations across the country. The sample for Survey 2 (using Tool 2) consisted of 317 students (second and third year undergraduates). Of these, 252 students were drawn from 4 local colleges and the remaining 65 were drawn from a large number of colleges and institutes at 25 locations in the country, who attended a summer enrichment program (unrelated to the present work) at our institution. Some additional points regarding the two samples may be noted: (i) Surveys 1 and 2 had no common student, (ii) Survey 2 had no first year undergraduates, (iii) Survey 2 has smaller size than Survey 1 but greater diversity in the number of colleges or institutes across the country, and (iv) Survey 2 had a greater number of meritorious students (see Sec. III B). The yearwise distribution of students in the two samples is given in Table I.

No formal statistical sampling procedure was followed in either survey. The choice of the college or institute was based simply on our reach, the willingness of the college, and other logistics. However, both surveys ensured that the samples were drawn from two somewhat distinct types of institutions, local colleges affiliated to a university, and autonomous national institutes of excellence, or reputed colleges. For data analysis, however, we disregard this category.
TABLE I. Sample distribution.

\begin{tabular}{lrc}
\hline \hline Year of study & Survey 1 & Survey 2 \\
\hline First year & 34 & $\ldots$ \\
Second year & 345 & 242 \\
Third year & 115 & 75 \\
Unknown & 10 & $\ldots$ \\
Total & 504 & 317 \\
\hline \hline
\end{tabular}

\section{E. Reliability}

The usual split-half method was used for testing the reliability of the tool. The questionnaire was split into two equal parts such that there was one-to-one correspondence between propositions in the two parts with common epistemic category. Table II gives the values of the reliability coefficient $R=2 r /(1+r) \quad(r$ being the Pearson correlation coefficient) [13] for the two surveys.

Since the questionnaire measures the epistemic understanding in terms of three broad constructs (nominal, physical, and mathematical warrants), we also compute the Cronbach's $\alpha$ for each of these three classes. The results of these tests, given in Table II, demonstrate the overall reliability as well as internal consistency of the items belonging to each construct separately.

\section{F. Discrimination index}

Table II also gives the discrimination index $D$ of the aggregated items belonging to the three classes (nominal, physical, mathematical) for the two surveys. The discrimination indices are good for each class for both surveys. Interestingly, the index improves in each class from Survey 1 to Survey 2; markedly so for the nominal class.

\section{RESULTS}

Figure 1 gives the overall distribution of epistemic scores $\left(S_{E}\right)$ in the full sample for the two surveys. For Survey 1 (2), the mean epistemic score was 33.8 (48.0) and the median was 28.0 (41.7) out of a maximum possible score of 100. It is clear that on the whole, physics students have poor epistemic clarity in the sense we have defined the term.

TABLE II. Reliability coefficient, Cronbach's $\alpha$ and discrimination index $(D)$.

\begin{tabular}{lcc}
\hline \hline & Survey 1 & Survey 2 \\
\hline Reliability $(R)$ & 0.89 & 0.92 \\
$\alpha$ (Nominal) & 0.77 & 0.85 \\
$\alpha$ (Physical) & 0.73 & 0.80 \\
$\alpha$ (Mathematical) & 0.83 & 0.86 \\
$D$ (Nominal) & 0.43 & 0.66 \\
$D$ (Physical) & 0.44 & 0.49 \\
$D$ (Mathematical) & 0.58 & 0.64 \\
\hline \hline
\end{tabular}




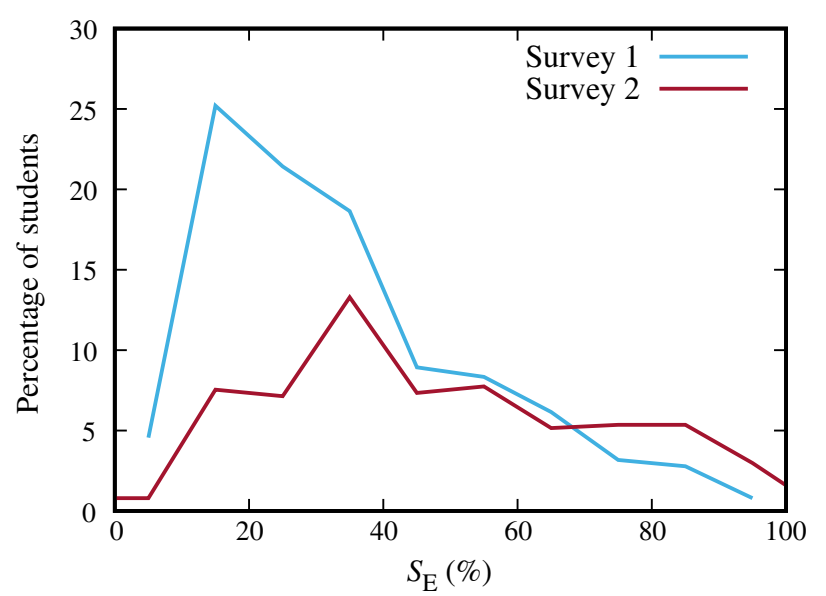

FIG. 1. Distribution of percentage epistemic scores $\left(S_{E}\right)$ of students.

These measures show a marked increase in the performance from Survey 1 to Survey 2. This, however, is a combined effect of two factors: (i) the higher overall merit (in terms of academic marks) of students in Survey 2, and (ii) the attempted disambiguation of epistemic categories in Tool 2, brought about by the appended orientation note. The effect of the second factor alone emerges in Sec. III B.

We next study how the epistemic scores are related to the exposure of the students to physics, and to their performance in standard tests of content knowledge. To do this, the year of study at the undergraduate level of the respondents and their academic marks, which were part of the information gathered in the tool, were chosen as the category variables in our analysis. Gender was not a category variable.

\section{A. Epistemic score and year of study}

An ANOVA test for Survey 1 revealed no group with significantly different epistemic scores across the three years of study ( $p=0.078$ ). The results of the $t$ tests (with Bonferroni corrections) across the different years of study in the two surveys are given in Table III. It is clear that there is no statistically significant difference between the epistemic scores of the students in different years in either survey. (This is especially so for Survey 2.) Thus in the following analysis we have merged the data of all students irrespective of their year of study.

TABLE III. Pairwise $t$ tests between epistemic scores of students grouped by year of study.

\begin{tabular}{llcccc}
\hline \hline Survey & Subsamples & $t$ & $t_{\text {crit }}$ & $p$ value & Inference \\
\hline 1 & I and II year & 1.78 & 3.40 & 0.08 & Insignificant \\
& II and III year & 0.95 & 3.19 & 0.32 & Insignificant \\
& I and III year & 1.29 & 3.36 & 0.20 & Insignificant \\
2 & II and III year & 0.13 & 3.08 & 0.90 & Insignificant \\
\hline \hline
\end{tabular}

\section{B. Epistemic score and content knowledge}

As mentioned earlier, our study did not include a separate test of content knowledge; instead, we use the "academic marks" of the students as an indicator of content knowledge. By academic marks in physics $\left(M_{P}\right)$ and mathematics $\left(M_{M}\right)$ we mean the scores at the Higher Secondary (high school) Board examination in these subjects before entry to college or institute. However, in the collection of data, a fraction of the students did not report their academic marks. Thus the sample sizes for the two surveys for the purpose of this section only are 353 and 282, respectively. For the remaining analysis, the full samples have been used.

The Pearson correlation coefficients were determined to be 0.57 and 0.63 between $M_{P}$ and $S_{E}$, for Survey 1 and Survey 2, respectively. Between $M_{M}$ and $S_{E}$, these values were 0.50 and 0.53 , respectively. The correlation between $M_{P}$ and $M_{M}$ is, as expected, quite good ( 0.77 for Survey 1 and 0.84 for Survey 2). Therefore, in the following analysis, for simplicity we take $M_{P}$ only as the index of academic performance. The average value of $M_{P}$ and its standard deviation are 73.0 and 15.8, respectively, for Survey 1, and 77.8 and 17.1 for Survey 2.

To see why the degree of correlation between content familiarity and epistemic ability is reasonably good but still not very high, we investigate the data in more detail. We divide the entire sample into four groups of equal intervals of marks in physics: A $\left(M_{P} \geq 85\right)$, B $\left(70 \leq M_{P}<85\right)$, $\mathrm{C}\left(55 \leq M_{P}<70\right)$, and $\mathrm{D}\left(35 \leq M_{P}<55\right)$. The last one has been stretched slightly to accommodate the minimum passing marks in our system. The sample size and the average epistemic score for each group in the two surveys are shown in Table IV.

ANOVA tests yielded very small $p$ values $\left(7.3 \times 10^{-36}\right.$ for Survey 1 and $7.4 \times 10^{-28}$ for Survey 2), clearly showing the existence of at least one group with epistemic scores significantly different from the rest in each survey. This was followed by pairwise $t$ tests, including Bonferroni correction, whose results are given in Table $\mathrm{V}$. We see that in both surveys the top three groups, A, B, and C, have significantly different epistemic scores from each other, while the groups $\mathrm{C}$ and $\mathrm{D}$ do not have such a difference. We also computed Cohen's $d$ for each pair of groups to check for the effect size. The values listed in Table V indicate that the

TABLE IV. Sample size $(N)$ and average epistemic scores $\left(\left\langle S_{E}\right\rangle\right)$ of the groups A,B,C, and D.

\begin{tabular}{|c|c|c|c|c|}
\hline \multirow[b]{2}{*}{ Groups } & \multicolumn{2}{|c|}{ Survey 1} & \multicolumn{2}{|c|}{ Survey 2} \\
\hline & $N$ & $\left\langle S_{E}\right\rangle$ & $N$ & $\left\langle S_{E}\right\rangle$ \\
\hline A & 101 & 56.3 & 131 & 64.5 \\
\hline B & 102 & 32.8 & 50 & 46.1 \\
\hline $\mathrm{C}$ & 105 & 27.5 & 69 & 33.6 \\
\hline D & 45 & 22.8 & 32 & 29.7 \\
\hline
\end{tabular}


TABLE V. Pairwise $t$ tests and Cohen's $d$ between epistemic scores of students grouped by academic marks.

\begin{tabular}{lccccccc}
\hline \hline Survey & Subsamples & $t$ & $t_{\text {crit }}$ & $p$ value & Inference & Cohen's $d$ & Effect size \\
\hline 1 & A and B & 9.00 & 2.43 & $3.2 \times 10^{-16}$ & Significant & 1.07 & Very large \\
& B and C & 2.59 & 2.43 & $1.0 \times 10^{-2}$ & Significant & 0.36 & Medium \\
& C and D & 2.25 & 2.45 & $2.6 \times 10^{-2}$ & Insignificant & 0.36 & Medium \\
2 & & & & & & \\
& A and B & 5.50 & 2.45 & $3.4 \times 10^{-7}$ & Significant & 0.84 & Large \\
& B and C & 3.63 & 2.45 & $6.4 \times 10^{-4}$ & Significant & 0.66 & Large \\
& C and D & 1.00 & 2.49 & $3.2 \times 10^{-1}$ & Insignificant & 0.23 & Small \\
\hline \hline
\end{tabular}

difference between $\mathrm{A}$ and $\mathrm{B}$ has a very high level of significance in both surveys. This suggests that epistemic ability rises significantly with content knowledge only after some reasonable threshold of the latter-an important insight from the data.

Another notable finding emerges when we look at the scatter plots of the epistemic scores $\left(S_{E}\right)$ against $M_{P}$, for both surveys in Fig. 2. They show that high epistemic scores necessarily imply high academic marks which affirms in a sense the content validity of the tool we have developed. But the converse is not true; that is, several students with high academic marks exhibit poor epistemic scores. This finding is not entirely surprising and, in fact, reflects the well-known inadequacy of the country's examination system which enables students to score well in their board examinations even with poor content clarity.

Finally, for each epistemic category, we give plots of student responses in respect of both surveys for inspection in Fig. 3. The plots suggest qualitatively a broad similarity of responses. We note, however, that the different test statistics (reliability, Cronbach's $\alpha$, discrimination index, correlation between $S_{E}$ and $M_{P}$, average epistemic scores across different groups) for Survey 2 are somewhat better than for Survey 1, though along the same pattern. Also, importantly, the average epistemic score of each group (A, B, C, D) is higher in Survey 2 than in Survey 1 (see Table IV). Since each group consists of students of similar merit (in terms of $M_{P}$ ) in both surveys, this effect seems to be independent of the overall merit of the students. To check this effect properly, we carried out an ANCOVA on the $S_{E}$ with the two surveys as independent variables and $M_{P}$ as a covariate. For this comparison, only second and third year students were included, since Survey 2 had no first year students. Controlling for $M_{P}$, we obtain a $p$ value of $10^{-10}$ in the ANCOVA, confirming the inference that the epistemic scores in Survey 2 are distinctly better compared to those of Survey 1 . This is hardly surprising, since Tool 2 was aimed to minimize the ambiguity in the meanings of different epistemic categories. We confine our discussion henceforth to the results of Survey 2.

\section{Patterns and trends in epistemic errors}

To unravel significant patterns in student epistemic errors revealed in Survey 2, we examined data for each epistemic category (epistemic warrant). This is done by aggregating responses to all propositions corresponding to the particular epistemic category. Bimodal and even trimodal distributions are particularly interesting to look at since they bring out the pitfalls more clearly. To examine if a pitfall is robust or a good discriminator, we carry out the analysis by considering students in three contiguous groups of equal intervals in terms of their absolute percentage epistemic scores: 0 to $33.33,33.33$ to 66.67 , and 66.67 to 100 of
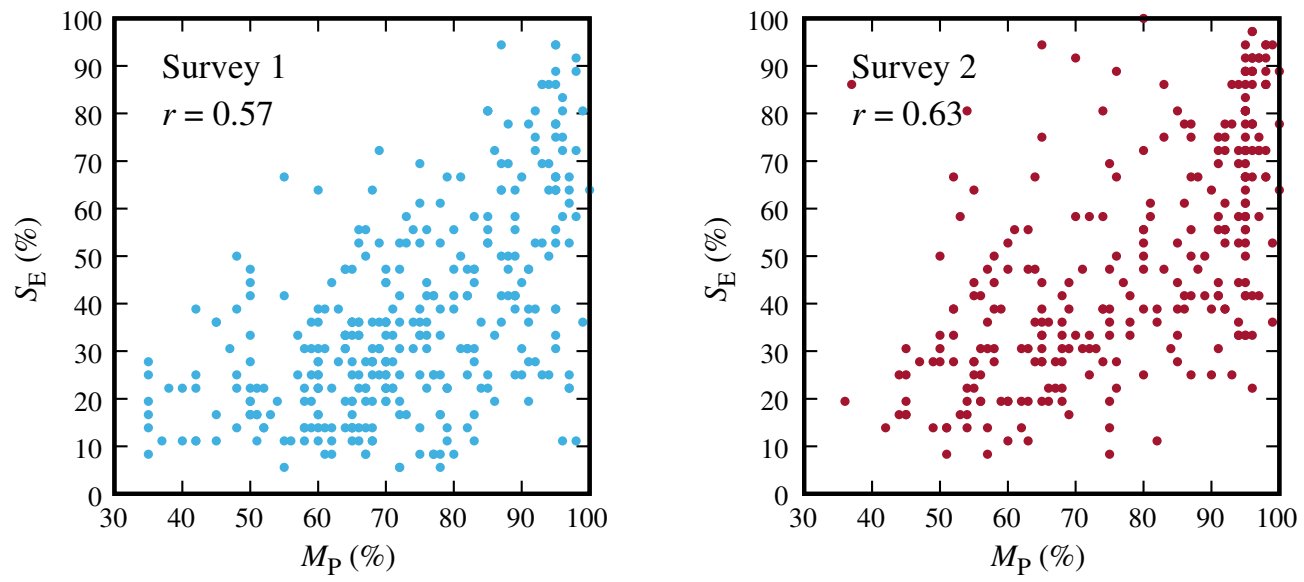

FIG. 2. Scatter plots of epistemic scores $\left(S_{E}\right)$ vs Higher Secondary Board examination marks in physics $\left(M_{P}\right)$ (Survey 1 in left panel, Survey 2 in right panel). The Pearson correlation coefficient $r$ is indicated in each case. 

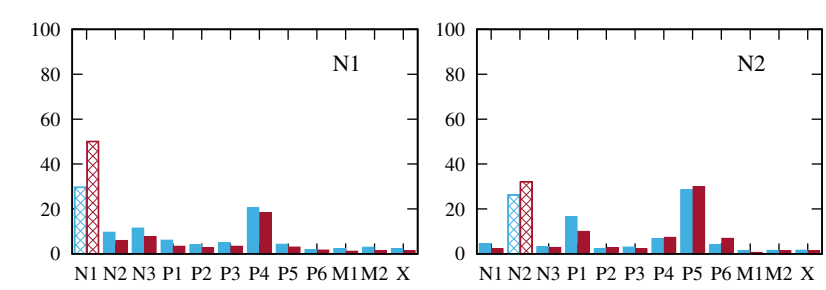

TABLE VI. Discrimination index $(D)$ of propositions and epistemic categories.

\begin{tabular}{|c|c|c|}
\hline Category & Proposition & $D$ \\
\hline N1 & $\begin{array}{c}\text { S5 } \\
\text { S7 } \\
\text { S8 } \\
\text { S9 } \\
\text { S13 } \\
\text { S17 } \\
\text { S19 } \\
\text { All }\end{array}$ & $\begin{array}{l}0.68 \\
0.76 \\
0.70 \\
0.65 \\
0.59 \\
0.75 \\
0.79 \\
0.70\end{array}$ \\
\hline $\mathrm{N} 2$ & $\begin{array}{c}\text { S1 } \\
\text { S10 } \\
\text { S28 } \\
\text { All }\end{array}$ & $\begin{array}{l}0.43 \\
0.63 \\
0.66 \\
0.57\end{array}$ \\
\hline M1 & $\mathrm{S} 23$ & 0.53 \\
\hline M2 & $\begin{array}{l}\text { S12 } \\
\text { S16 } \\
\text { S21 } \\
\text { S26 } \\
\text { S30 } \\
\text { S31 } \\
\text { S33 } \\
\text { S34 } \\
\text { S36 } \\
\text { All }\end{array}$ & $\begin{array}{l}0.57 \\
0.71 \\
0.53 \\
0.66 \\
0.62 \\
0.77 \\
0.68 \\
0.63 \\
0.68 \\
0.65\end{array}$ \\
\hline Category & Proposition & $D$ \\
\hline $\mathrm{P} 1$ & $\begin{array}{l}\text { S15 } \\
\text { S18 } \\
\text { S22 } \\
\text { S27 } \\
\text { All }\end{array}$ & $\begin{array}{l}0.65 \\
0.66 \\
0.67 \\
0.64 \\
0.66\end{array}$ \\
\hline $\mathrm{P} 2$ & $\begin{array}{l}\text { S4 } \\
\text { S6 } \\
\text { All }\end{array}$ & $\begin{array}{l}0.52 \\
0.55 \\
0.53\end{array}$ \\
\hline P3 & $\mathrm{S} 20$ & 0.44 \\
\hline P4 & $\begin{array}{c}\text { S2 } \\
\text { S3 } \\
\text { S11 } \\
\text { S25 } \\
\text { S29 } \\
\text { All }\end{array}$ & $\begin{array}{l}0.38 \\
0.28 \\
0.58 \\
0.53 \\
0.36 \\
0.42\end{array}$ \\
\hline P5 & $\begin{array}{l}\text { S14 } \\
\text { S24 } \\
\text { S32 } \\
\text { All }\end{array}$ & $\begin{array}{l}0.27 \\
0.28 \\
0.58 \\
0.38\end{array}$ \\
\hline P6 & S35 & 0.54 \\
\hline
\end{tabular}

FIG. 3. Aggregate distribution of responses to all propositions corresponding to each epistemic category for both surveys. The percentage distributions for the whole samples are shown. The light blue bars correspond to Survey 1 while the red bars correspond to Survey 2. The correct response is marked with a hashed pattern while other responses are marked in solid. The case of no responses is shown as "X."

sample sizes 94,136 , and 87 , respectively. These groups are referred to here as GL (low), GM (medium), and GH (high), respectively.

The aggregate distribution of responses to the different propositions corresponding to each epistemic category are shown in Figs. 4-13 for the whole sample as well as for the three groups GH, GM, and GL. There was no proposition corresponding to the nominal category N3. We also investigate data on each individual proposition under a given epistemic category to see if the patterns of errors depend on the context of the question. Accordingly, we generate four histograms for each of the 36 propositions. 
proposition belonging to that category. These indices are given in Table VI.

We now present our observations and inferences for each epistemic category one by one. In the brackets below are given the serial numbers of the propositions in the questionnaire corresponding to the category under discussion.

\section{N1 [Denoting by symbols] (S5, S7, S8, S9, S13, S17, S19)}

Denoting a quantity by a mathematical symbol is equated to an assumption (P4) by nearly $20 \%$ of students (top left panel of Fig. 4); this confusion is common to both GM and GL but is rare in GH. The context dependence of the error (not seen in the aggregated Fig. 4) is interesting. It shows up little in S5, modestly in S9 and S13, but considerably more in S7, S8, S17, and S19. Note that there is a striking commonality in the latter group in that they all involve the generic symbol $\Delta$ followed by $t, V, K$. Does the greater erroneous response in this group of propositions arise because they seem to involve more complex symbolization than the others? We do not know. On the whole, this category is a good discriminator between $\mathrm{GH}$ and the rest.

This confusion perhaps arises from the careless way the word "assumption" is used in physics classrooms. It is not uncommon for instructors to say: "assume $r$ to be the radius of the sphere" instead of "denote the radius of the sphere by $r$," or an equivalent "let $r$ denote the radius of the sphere."

Interestingly, a considerable number of students has opted for N3: an arbitrary convention, in S7. This response

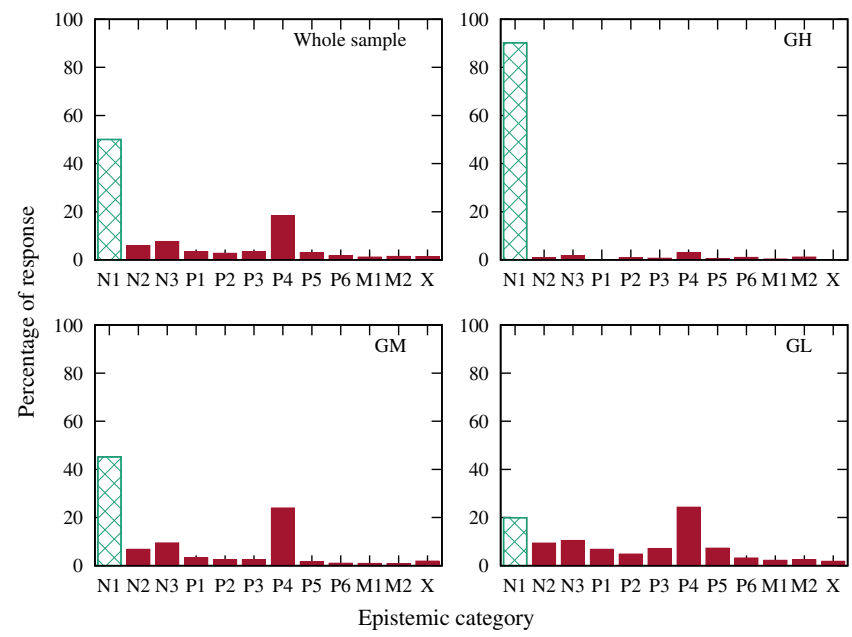

FIG. 4. Aggregate distribution of responses to all propositions (S5, S7, S8, S9, S13, S17, S19) corresponding to epistemic category N1. The percentage distributions for the whole sample, as well as the three groups, GH, GM, and GL are shown. The total for each subsample is normalized to $100 \%$. The correct response is marked with the hashed green pattern while other responses are marked in solid red. The case of no responses is shown as X. is seen to a large extent (33\%) in GL, a considerable extent in GM (26\%), and much less in GH (5\%); the likely reason being the use of the word "arbitrary" in S7. Perhaps, an alternative word such as "infinitesimal" would have avoided this error.

\section{N2 [Verbalization] (S1, S10, S28)}

This category turns out to show common errors across all groups; overall only $32 \%$ of students have correctly identified it. The graph is trimodal with considerable fractions of students opting for epistemic categories P5, and to a slightly lesser extent, P1. Naming of a property of a system (all three propositions S1, S10, and S28 refer to a property, not a physical quantity) is equated to an experimental fact (P5) or a definition (P1).

Giving a certain name to a physical quantity is clearly a linguistic choice, often governed by the closeness of the lay meaning of the chosen term in that language with the intended meaning in physics. However, though the latter meaning is precise, the meanings of words in a natural language are imprecise and context dependent. For example, the terms force, energy, impulse, momentum, etc., have distinct and precise meanings in physics, while they may be interchangeably used with roughly the same meaning in different contexts in the English language. In domains of physics far removed from lay contexts, the choice of terms is often completely arbitrary, such as the use of terms like color, flavor, charm, and numerous other terms in different areas of physics.

Thus, naming a physical quantity by a certain word (N2) is a choice and obviously has nothing to do with a physical fact or principle (P5). The same goes for naming of a property of a system or a process under some conditions. Here again the linguistic term used is often very apt and conveys the intended meaning fairly well. (It happens to be so in our propositions S1, S10, and S28 that use the words "steady," "incompressible," and "nonviscous," respectively.) Nevertheless, it is still a choice of a word and in no way can be regarded a fact or a principle of physics.

As for confusion between $\mathrm{N} 2$ and $\mathrm{P} 1$, these propositions can certainly be regarded as definitions of linguistic terms in physics but they are certainly not definitions of any physical quantity in terms of other physical quantities (P1). This confusion has led to a considerable fraction of GM and GL students to opt for P1.

Knowing the precise meaning of a technical term in physics is certainly a part of learning physics, which has the epistemological role of communication in the community. This knowledge belongs to the nominal category, distinct from the knowledge of quantitative definitions of physical variables in terms of others, which is an essential part of the content of physics. A change in the name of some technical term will not have implications in physics as such. On the other hand, there is not much flexibility in the definitions of 


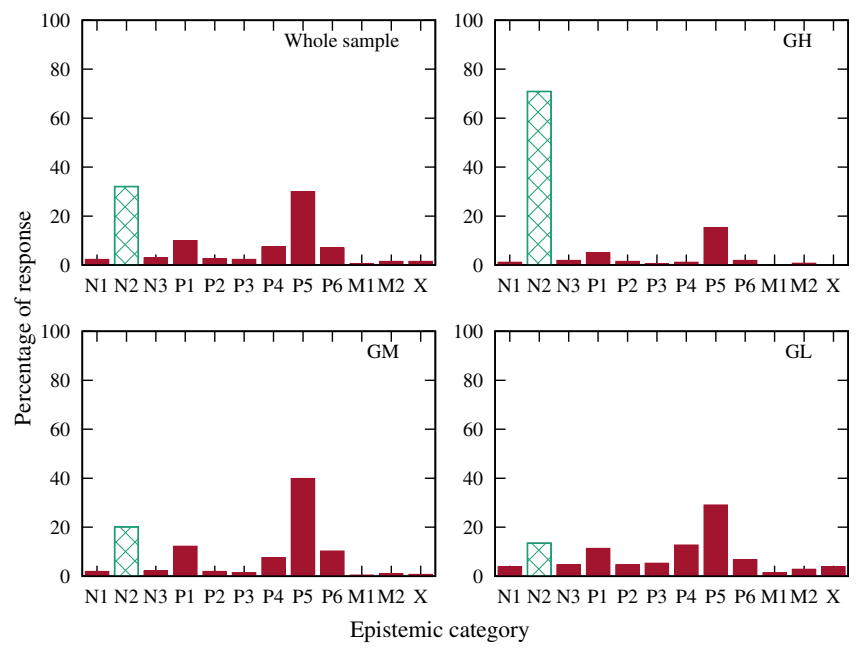

FIG. 5. Aggregate distribution of responses to all propositions (S1, S10, S28) corresponding to epistemic category N2. Format of the figure is the same as in Fig. 4.

physical quantities, except perhaps in overall constants. Any nontrivial change in the definitions (e.g., from Newtonian to relativistic definition of momentum and kinetic energy) has important physical implications.

\section{P1 [Physical definition] (S15, S18, S22, S27)}

The general trend under P1 is quite clear from Fig. 6. Students are prone to take mathematical definitions of physical quantities as mathematical formulas (M1), as seen most clearly for GM and GL. This is the familiar epistemic error of ascribing the warrant of any mathematical relation in physics to mathematics; the figure clearly shows this to be an excellent discriminator between $\mathrm{GH}$ and the rest. Not surprisingly, this pitfall is most stark in S22 which "looks" more mathematical as it involves an integral.

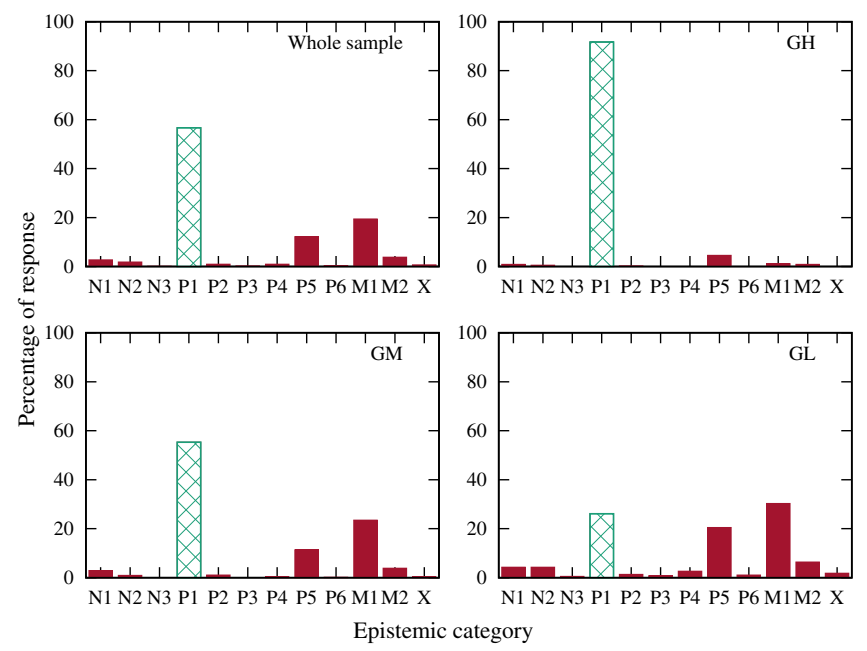

FIG. 6. Aggregate distribution of responses to all propositions (S15, S18, S22, S27) corresponding to epistemic category P1. Format of the figure is the same as in Fig. 4.
There is also confusion between a physical definition (P1) and a physical law (P5) which is more interesting. Again, it is more pronounced in GM and GL than in GH. Experience shows that there is lack of appreciation that the relations $K=\frac{1}{2} m u^{2}$ and $d W=\vec{F} \cdot \overrightarrow{d s}$ are definitions of quantities called kinetic energy and work, respectively. They are no doubt extremely useful definitions using that the scalar consequence of the second law of Newton gets expressed elegantly as the so-called work-energy theorem: $\Delta K=\int \vec{F} \cdot \overrightarrow{d S}$. Further, with this definition combined with a suitable definition of another physical quantity (potential energy), some basic forces in mechanics admit the principle of conservation of total mechanical energy of a particle in motion under such forces; and so on. Thus, though in principle a definition is arbitrary (there is nothing right or wrong about a definition), one generally would encounter definitions in physics that are useful. Nevertheless, a useful definition cannot be equated to a law.

Thus the observed confusion between P1 and P5, particularly among the lower groups of GM and GL, is certainly indicative of a lack of epistemic clarity. See, however, the discussion under Sec. III C 7.

\section{P2 [Choice without assumptions] $(S 4, S 6)$}

Data under this epistemic category (Fig. 7) show an interesting variation in response between the two propositions (S4 and S6) that correspond to it. In S4, where a choice of axes is involved, nearly $97 \%$ of GH are right as against $45 \%$ of GL. A small but non-negligible number of GM and GL has opted for P3 perhaps simply because of the word "figure" occurring in the proposition. The more significant pitfall is between choice (P2) and assumption (P4), which appears mainly in S6 across all groups; about $29 \%$ of even GH students reveal this pitfall in S6.

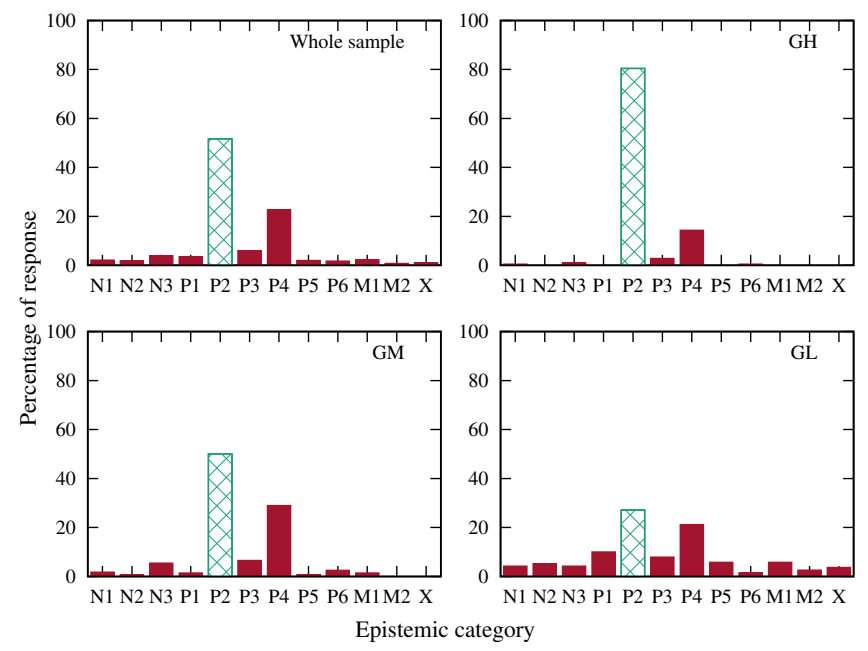

FIG. 7. Aggregate distribution of responses to all propositions (S4, S6) corresponding to epistemic category P2. Format of the figure is the same as in Fig. 4. 
Evidently, many students fail to appreciate that choice of axes, system, etc., amounts to no loss of generality in the given derivation, i.e., there is no physical assumption involved therein.

One aspect of the orientation note in Tool 2 was to eliminate the ambiguity between P2 (choice) and N3 (convention). A convention is no doubt a choice, but its purpose is only to establish a common usage in the community. $\mathrm{P} 2$, on the other hand, is a nontrivial choice for simplification of treatment of a problem that does not entail any assumption. It is context dependent. That the polar axis in spherical polar coordinates is always taken to be the Cartesian $z$ axis is a convention (N3); with this convention, in a problem in which there is rotational symmetry about a direction, taking the $z$ axis along that direction is a choice, one that simplifies the treatment (P2). A sensible choice of system, frame or axes, etc., is part of the expert skill set; on the other hand, using a different convention has no bearing on the treatment of a problem. As expected, data show a significant drop in the extent of N3 response for P2 from Survey 1 to Survey 2.

\section{P3 [Diagram based reasoning] (S20)}

Proposition S20 in our tool illustrates this new category that we discussed in Sec. II A. To convert our derivation in pure algebraic terms without reference to a figure may be possible but will be needlessly tedious.

Our data, shown in Fig. 8, are as expected. Because of the phrases "from figure" and "darker shaded portions" in the proposition, the overall performance is good, with 93\% of $\mathrm{GH}, 75 \%$ of GM, and $49 \%$ of GL giving the correct response. The orientation note states that $\mathrm{P} 3$ is not a mere reference to a figure but is about reasoning based on a figure, as distinct from algebraic reasoning. The distinction is subtle and may be the reason why about half of GL

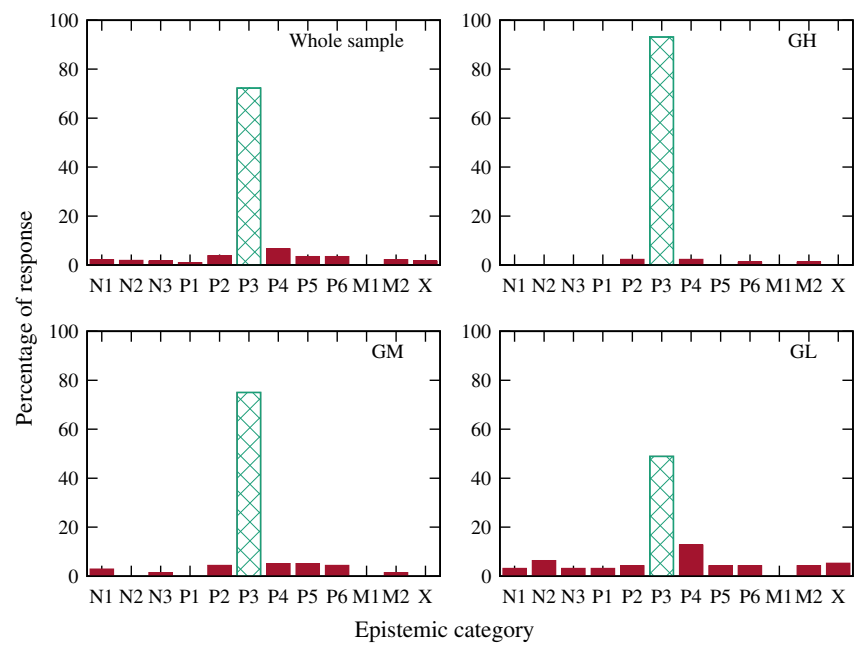

FIG. 8. Aggregate distribution of responses to the only proposition (S20) corresponding to epistemic category P3. Format of the figure is the same as in Fig. 4. students have not opted for P3, which involves both a reference to the figure and an argument based on it.

\section{P4 [Assumptions or approximations] (S2, S3, S11, S25, S29)}

This category turns out not to be a good discriminator, partly because of considerable commonality of the errors across the groups, and partly due to the fact that some propositions are relatively easy to classify. Data under this epistemic category, shown in Fig. 9, reveal interesting mixups between P4 and P2, P3, P5. There is considerable context dependence as evident in the detailed histograms for individual propositions (given in Figs. 19-28 in the Supplemental Material [14]).

The proposition S2 ("we consider the situation when the fluid flow is steady"), an assumption from our point of view, is regarded as a choice of a fluid in steady flow by more than $10 \%$ students across all the groups. Logically, an assumption regarding a system can always be termed a choice of the corresponding idealized system. What has not been realized is that P2 is a choice that does not entail any physical assumption, while $\mathrm{P} 4$ does, as has been made clear in the orientation note.

In S3 ("the parts of the tube near ends A and B are horizontal") the P4-P2 mix up continues, but the more remarkable feature there is a $\mathrm{P} 4-\mathrm{P} 3$ mix up. This is clearly due to a reference to a diagram in S3. The orientation note makes it clear that $\mathrm{P} 4$ includes assumptions regarding both text and diagram, while P3 involves reasoning based on a diagram, not a mere reference to it. Data show that this distinction has not been internalized by many students across the groups ( $\mathrm{S} 3$ has poor discrimination index).

In S25 (" $g$ is taken to be a constant"), we find a P4-P5 confusion. Students consider this statement to be an experimental fact (P5), rather than an approximation

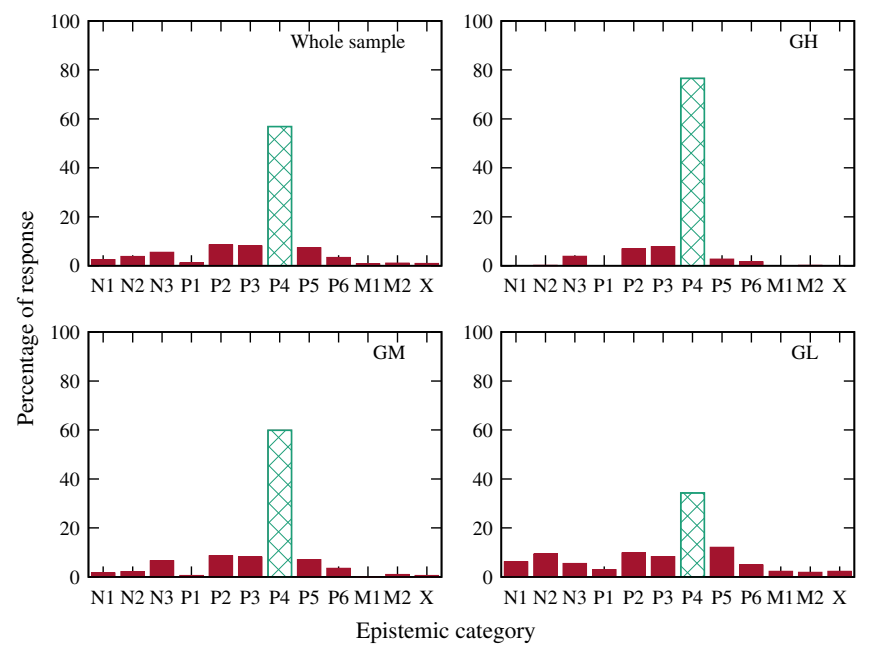

FIG. 9. Aggregate distribution of responses to all propositions (S2, S3, S11, S25, S29) corresponding to epistemic category P4. Format of the figure is the same as in Fig. 4. 
(P4), presumably due to the ubiquity of this approximation in introductory texts. Lastly, S29 ("we assume the fluid to be nonviscous") does not reveal anything significant; the overall performance being markedly higher, presumably due to the explicit use of the word "assume" in the proposition.

\section{P5 [Empirical law or measurement] (S14, S24, and S32)}

This epistemic category again turns out not to be a good discriminator, particularly for propositions S14 and S24 (see Table VI). Data in Fig. 10 suggest students conflate this category with $\mathrm{P} 1$ or $\mathrm{M} 2$ or even P6. There is interesting context dependence of these confusions.

The P5-P6 and P5-M2 confusions appear mainly in $\mathrm{S} 14$, and that too, surprisingly for $\mathrm{GH}$. We are unable to interpret the first, since S14 is so clearly the empirical fact of mass conservation. The second confusion is understandable since the proposition S14 involves both an empirical fact and its consequence in the given derivation. Thus, as per our scheme, it belongs to P5. Perhaps it would have been better if it were split into two propositions: one stating the mass conservation law (P5), and the other its logical deduction (M2). We realized this after Survey 1 , but chose not to correct this proposition in Survey 2, so as to be able to compare the two surveys that do not differ in the questionnaire, but only in the supplementary orientation note.

The P5-M2 confusion, in general, may arise due to the appearance of the word "deduction" in the phrasing of P5 also, when M2 already stands for logical or mathematical deduction. For example, we assign P5 to proposition S32, but someone could argue that it is not a law by itself but a mathematical deduction of Newton's second law and hence should belong to M2. The answer is that the correct option

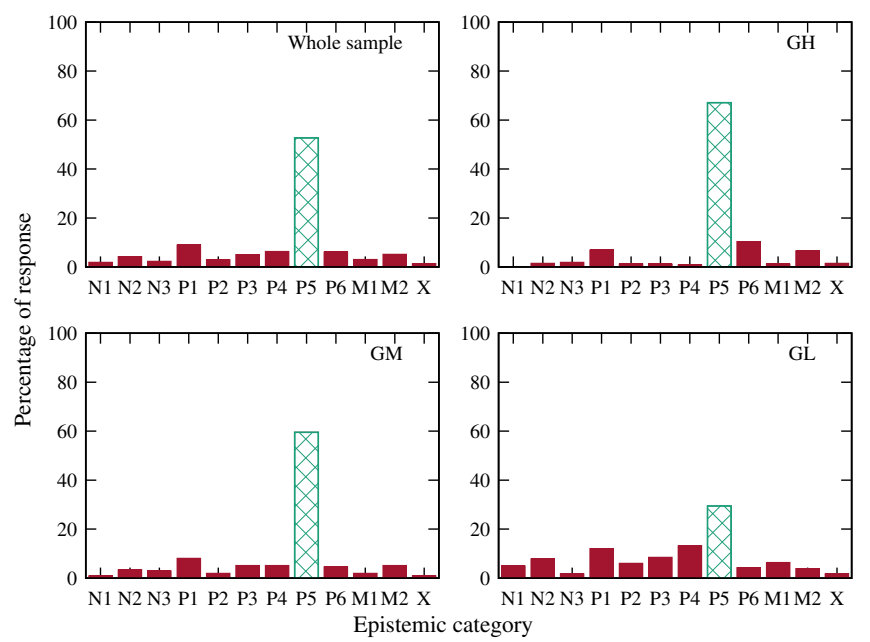

FIG. 10. Aggregate distribution of responses to all propositions (S14, S24, and S32) corresponding to epistemic category P5. Format of the figure is the same as in Fig. 4. is intended to be with reference to the given derivation. If in the derivation we had started with the second law and arrived at the work-energy theorem after a few mathematical steps with no further physical inputs, S32 would be M2 type. What is quoted in the present questionnaire is, however, a scalar version of the second law in terms of work and energy, and hence is P5 type. In anticipation of such ambiguity, in the actual administration of the questionnaire, students were asked to number the previous propositions relevant to M2 (though in scoring them, we disregarded whether or not they did so).

Lastly, the P5-P1 conflation reflects the same P1-P5 conflation seen earlier under P1. Again, it has a proposition dependence; it shows up markedly in S24, and hardly at all in S14 or S32. This is perhaps because the latter two are qualitative statements of physical principles, and students are unlikely to consider them as mathematical definitions of physical quantities (P1). On the other hand, S24 is a mathematical equation, expressing a quantity, namely, force as the product of two other physical quantities, $m$ and $g$. This misleads some students to consider it as $\mathrm{P} 1$, not realizing that it is, in fact, a statement of Newton's second law, and hence should fall under P5.

Of course, at the most foundational levels of physics, the P1-P5 conflation is rather subtle. Newton's discovery of the second law $\vec{F}=m \vec{a}$ may well be regarded as the discovery of an enormously useful definition of a physical quantity called force, one that becomes a testable law of physics when an explicit form of force is given (such as inverse-square gravitational force). Further, the definition is one for which the third law is valid in nature. It is, however, extremely unlikely that this epistemic ambiguity has in any way affected the student responses. Students at the college level are never exposed to these philosophical subtleties. In instruction as well as in textbooks, Newton's laws are always referred to as "laws" of motion, and not as definitions. Thus we interpret these data as revealing a pitfall rather than a deep philosophical disagreement.

\section{P6 [Heuristic reasoning] (S35)}

There is only one proposition (S35) belonging to this category. As remarked earlier, one of the experts insisted on assigning it to M2. The result derived in the questionnaire (S34), however, refers to the case where the ends of the tube are horizontal, as shown in the figure, amounting to a simplifying situation. Thus going from S34 to S35 is a conjectural jump, which means S35 cannot be regarded as a direct deduction (M2) in the given derivation. It is another matter that the generalization may be very simple to prove-in the given derivation it is an unproved though a very informed guess (P6).

Data show this proposition to be a good discriminator between GH and the rest. Nearly $67 \%$ of GH get it right, while both GM and GL show poor performance (about $29 \%$ and $13 \%$, respectively). The most marked pitfall 


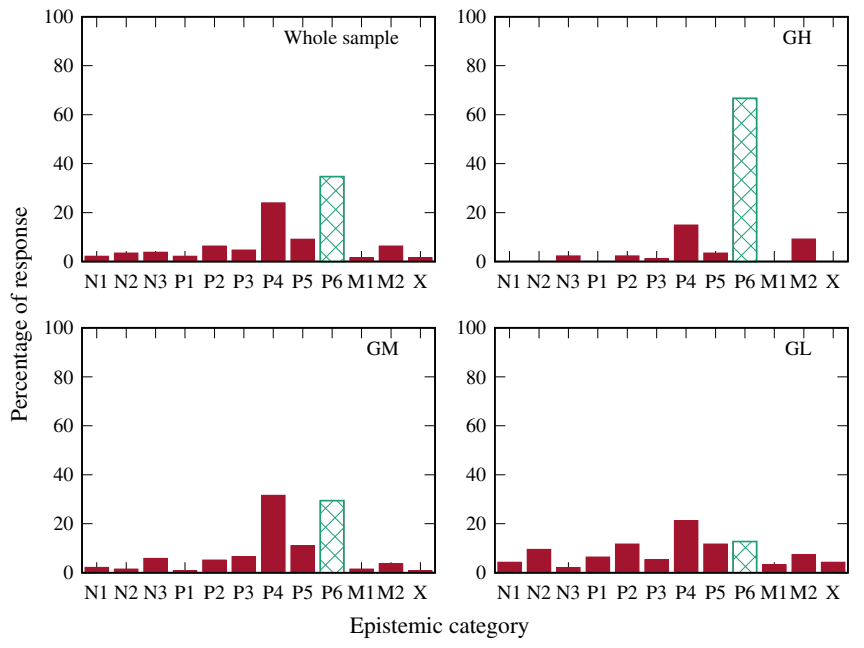

FIG. 11. Aggregate distribution of responses to the only proposition (S35) corresponding to epistemic category P6. Format of the figure is the same as in Fig. 4.

across all groups is to confuse $\mathrm{P} 6$ with physical assumption P4 (see Fig. 11), again possibly arising from the loose and undifferentiated use of the word assumption in physics instruction. (We saw this in the discussion under $\mathrm{N} 1$ also). In ordinary language we might call a guess an assumption, but in physics an unproved (if plausible) generalization of a result of a derivation is quite distinct from the physical assumptions made in arriving at that result in the given derivation.

\section{M1 [General mathematical result] (S23)}

Here again, only one proposition (S23) is M1 type and a good fraction of GH (80\%) and a much smaller fraction of GM (56\%) give the correct response (Fig. 12). The much

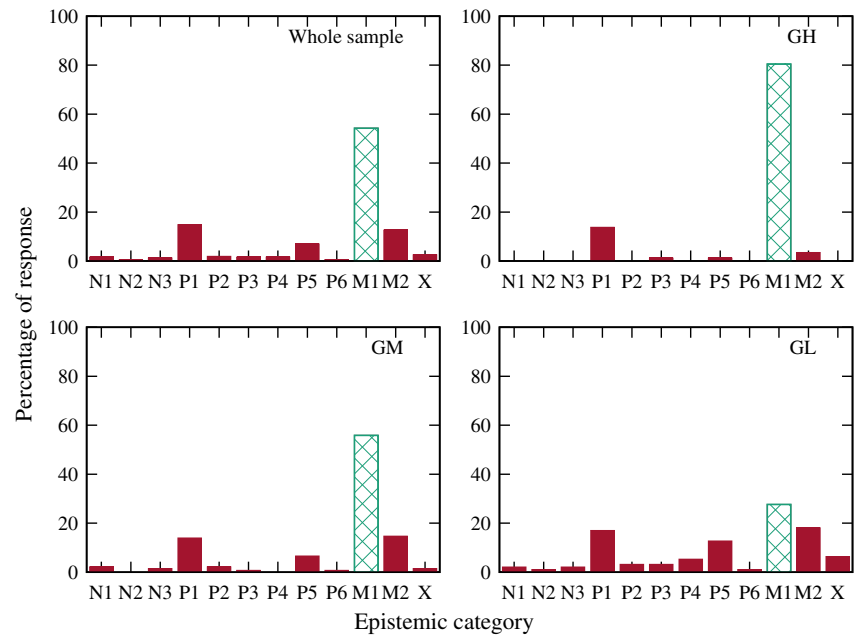

FIG. 12. Aggregate distribution of responses to the only proposition (S23) corresponding to epistemic category M1. Format of the figure is the same as in Fig. 4. poorer performance $(28 \%)$ of GL shows that a large number of undergraduate physics students fail to separate a contextindependent mathematical input from the rest of a derivation in physics.

\section{M2 [Deduction without further physical input] (S12, S16, S21, S26, S30, S31, S33, S34, S36)}

A total of 9 propositions belong to M2, typical of any derivation in physics, which consists in the main of mathematical or logical deductions based on a few physical and mathematical propositions. As mentioned earlier, students were instructed to indicate these preceding propositions. However, only a negligible fraction of them actually did so. A correct identification of all preceding propositions needs considerable diligence that we did not wish to test at all. The instruction was merely meant to avoid confusion between M2 and P5 which also uses the word deduction. Accordingly, our grading did not take into account whether they identified the preceding propositions, and if so, correctly or not. No analysis was, therefore, carried out on this aspect.

This category turns out to be a clear discriminator between GH (76\%), GM (33\%), and GL (11\%) in the combined data as seen in Fig. 13. One intriguing feature that emerges is that a considerable fraction of students (19\%) choose P1 (mathematical definition of physical quantity) for an M2 proposition. A propositionwise analysis shows that this is strongest in S21, and considerable in S26, S31, and S33. In fact, this mix-up shows up for GH students markedly (37\%) only in S21. This proposition has one term on the left-hand side that equals a mathematical expression involving several other variables on the righthand side, somewhat akin to a mathematical definition of a physical quantity. Thus students unable to identify this as a

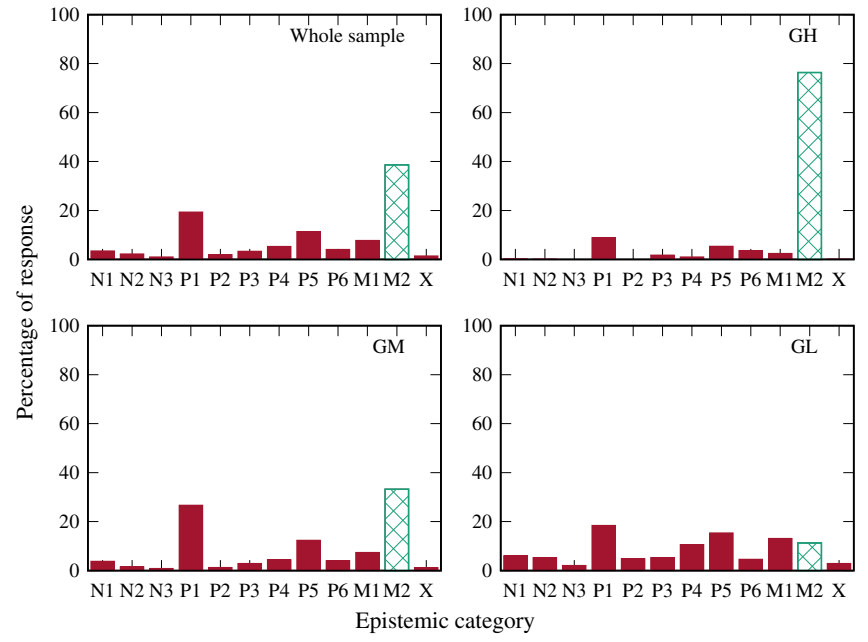

FIG. 13. Aggregate distribution of responses to all propositions (S12, S16, S21, S26, S30, S31, S33, S34, S36) corresponding to epistemic category M2. Format of the figure is the same as in Fig. 4. 
deduction from previous steps seem to fall for the choice of P1. Somewhat similar structures of the propositions S26, S31, and S33 seem to lead GM and GL students to the same error.

On the whole, a natural way to interpret the data seems to be that the meritorious GH students have greater mathematical competence than others to be able to see several steps in the derivation as straightforward mathematical deductions with no other inputs, which students with inadequate competence in mathematics cannot see or are not confident about. The lowest performance across all groups occurs for S21 and S30, both of which involve deduction based on several preceding propositions.

\section{Summary of epistemic errors}

Ignoring the details and nuances, some broad takeaways from the above observations on epistemic errors are that a considerable fraction of physics undergraduates (i) cannot comprehend a nominal proposition for what it is: empty of any physics or mathematics, but rather equate it to a physical definition or even a physical law; (ii) are unable to distinguish between a choice without loss of generality and an assumption or approximation; (iii) do not seem to appreciate diagram-based reasoning as a distinct mode of reasoning and not a mere reference to a diagram; (iv) equate a physical definition to a law; (v) are unable to tell apart a result that has been proved in the derivation from one that has been only heuristically generalized; (vi) are unable to identify a contextindependent mathematical result from the maze of mathematical steps in a derivation; (vii) are unable to see that a certain result in a derivation is a straightforward mathematical deduction that follows from some earlier steps without any further physical input.

A few of these errors, e.g., (i) and (ii), are somewhat common across all groups of students, while others show good discrimination between the meritorious from the rest. However, there is much context dependence seen in the data; the extents of the errors and the discrimination between the groups depend on the proposition in question.

Lastly, we note a few imperfections of the tool itself revealed by our reflection on data as well as by reviewers. These will be corrected in future refinement of the tool. A proposition $(\vec{A} \cdot \vec{d} s=d V$ for an infinitesimal cylinder, right circular or oblique) should be quoted as a mathematical formula (M1) before S30 to make it M2 and remove its ambiguity with P3. Proposition S14 should be split into two propositions: mass of a fluid is conserved (P5); the mass of the fluid leaving end $\mathrm{A}$ and the mass of the fluid leaving end $\mathrm{B}$ in time $\Delta t$ are equal. The orientation note should include examples to clarify diagrammatic reasoning (P3), and also clarify arbitrary nominal convention (N3) from other nominal categories (N1, N2) as well as physical choice (P2).

\section{DISCUSSION}

This work is an elaboration of a new approach [8] that attempts to measure epistemic understanding of college physics students using a novel tool, which though content based, is altogether different from the familiar concept inventories in different topics. It derives from the feeling that the ability to discriminate between different epistemic warrants for propositions in a mathematical derivation in physics is important for its proper understanding. Of course, this is just one aspect of understanding a derivation; equally, and perhaps more important, is the understanding of the concepts, their distinctions, and interconnections as well as the place of a derivation in the subject. Also, there are several other aspects of content-based epistemic understanding of physics that this tool does not test, such as the knowledge of the structure and organization of the subject, the consistency among its different parts, the basic categories of its concepts, a feel about which law is more basic, which is more phenomenological, which is more stable, and which is more fluid, norms and practices of creating and validating new knowledge of physics, and many other things.

The standard measures of reliability of the tool, its internal consistency for each epistemic class (nominal, physical, and mathematical) and the discrimination index are all seen to be quite satisfactory (Sec. II). A facilitating aspect of the present tool is that though it is content based, it does not place any demands on the student's prior familiarity with the mathematics or physics of the derivation, since it is spelled out in detail as part of the tool itself.

However, there are two key questions regarding this work. First, is the tool measuring what it purports to measure? Second, is this work of any educational relevance? We examine these matters below.

\section{A. Construct validity}

Several issues threaten the construct validity of our tool: (i) expert validation, (ii) the effect of nonepistemic confounding variables on the epistemic measure, and most importantly, (iii) the distinction between learning our epistemic coding scheme correctly and genuine contentbased epistemic understanding.

We have already mentioned that expert validation of our tool was not perfect. Indeed, it seems unlikely that our approach can yield a tool that has total and unequivocal expert agreement that concept-based tests do. Our categorization does not have rigorous justification in philosophy or linguistics. These are simply sensible and useful categories in the limited context of physics education. The orientation note in the revised tool is an attempt at disambiguation of categories and conveying their intended meanings as clearly as possible. Yet, there could be a few propositions where experts might differ among themselves. This can arise due to a number of reasons. 
First, there might be a few minor imperfections in the tool as noted at the end of Sec. III. These can, of course, be corrected in a revised version of the tool. Second, a proposition that we assign to $\mathrm{P} 2$ (choice without assumption) could turn out to entail an assumption at a deeper level, far beyond the scope of the student. For example, the freedom in the choice of origin of coordinates and the orientation of the axes may, at a fundamental level, be related to the assumption of homogeneity and isotropy of space. However, in the present context, it is inconceivable that an expert would regard a proposition based on such a choice (e.g., S4) as P4 (assumption) instead of P2. Third, at the foundational level it is well known that definitions and basic laws are inseparably intertwined. In the context of the present tool, this could lead to P1-P5 conflation, for example, in S24, as discussed in some detail in Sec. III C 7. Lastly, the new category of diagram-based reasoning again can have expert disagreement. We have distinguished reasoning based on a diagram (that is difficult to substitute by algebraic reasoning) from a reasoning that uses a diagram drawn only for facilitation. This difference is not unambiguous in some propositions (e.g., S30).

The tool partly addresses this anticipated problem by asking to choose the most relevant (instead of the correct) code, when two choices may both be sensible but this should be more explicitly stated in the instructions. In any case, some residual expert disagreement is quite possible, indeed likely, in tools of this kind even after we refine and reword the categories. A possible way out is to slightly modify our scoring key to accommodate acceptable difference in expert responses to some of the propositions. This, however, needs to be thought through carefully in a future work.

Another concern is whether the measure of epistemic ability obtained from our tool is seriously confounded by other variables like diligence, memory (long term and working), language comprehension (of non-native speakers), etc., required in going through our coding scheme. We tried to reduce somewhat the effect of working memory by listing our codes at the bottom of each page of the questionnaire. (This is suppressed in the paper for brevity.) As for language comprehension, the entire sample was drawn mainly from urban colleges and institutes where comprehension of written English is reasonable, if not perfect. Yet, there is no doubt that the epistemic score must have been affected by these nonepistemic variables. Our data show good correlation between epistemic scores and academic marks in standard public examinations ( 0.53 for math and 0.63 in physics). Might this not arise primarily because these other variables are common to both?

This point needs careful consideration. First note that there is good but not excellent correlation between the scores. This is best seen by looking at the scatter diagrams in Fig. 2, which show that high epistemic scores necessarily imply high academic marks, but the converse is not true.
This feature lends an important clue to the interpretation of the observed correlation.

It is well known that the public board examination system of the country tests diligence and memory, language comprehension, etc., far more than genuine content understanding $[15,16]$. Thus students with high academic marks represent a mixed group in terms of content clarity (high, medium, and low). If our questionnaire were to measure primarily the nonepistemic variables mentioned above, one would expect all students with high academic marks to achieve high epistemic scores; but this is not the case. The scatter diagram is best interpreted by positing that the epistemic score does measure something not tested in the standard examinations, in addition to measuring other nonepistemic variables.

Finally, even if one grants that the tool is measuring something apart from the common variables mentioned above, the important issue is: just what is it measuring? It is possible to infer from our data that this "something" is partly the students' learning of the formal codes and the common terms used by physicists to describe them, rather than any deep understanding of epistemic distinctions, much less general content-based epistemic understanding described in the introductory para of this section. Thus, what we call epistemic errors could partly be just errors in learning the coding scheme and its language. Only detailed clinical interviews which probe the reason behind the students' choice (not carried out in the present work) can perhaps shed light on this issue.

To summarize, despite the numerous interesting features of our data on errors and correlations described in Sec. III, it is not possible to claim construct validity of our tool in a convincing way. However, our approach can be educationally relevant, even if the tool does not fully capture the construct "epistemic understanding." What it is probing, namely, the ability to discriminate between our epistemic categories, could still be educationally important.

\section{B. Pedagogic use}

As mentioned before (Sec. I), the epistemic categories adopted here are not intended for philosophical analysis. They are in a way common sense categories which any expert or teacher uses in understanding a chained argument in physics. It is not uncommon to hear from a teacher in a classroom phrases like "from this step onwards, it is just algebra," or "for this, we use a theorem from calculus," or "we will neglect such and such effect to simplify the problem," or "this property is known as inertia," or, "now using the invariance of this, or conservation of that, we get...", and so on. The expert implicitly knows the epistemic status of these different propositions, but may not always highlight their epistemic discrimination during teaching, and, occasionally, may be careless about correct usage ("assume" for "denote" or "choose") or may not 
highlight the distinction between an assumption entailing an approximation from a choice without loss of generality.

On the other hand, data from fairly large and diverse samples in our work show that a good many students are not clear about these epistemic distinctions and reveal interesting common errors. That these are not random mistakes is indicated by two features; first, two entirely different samples reveal a similar pattern of errors. They show the same primary, secondary, and tertiary peaks (see Fig. 3) with the expected difference that Tool 2 gives slightly higher scores due to the accompanying orientation note. Second, several of them seem to be "reasonable" (or better, expected) epistemic errors, much as alternative conceptions often have a reasonableness about them.

Still, there can be a question if we should worry about these errors and whether students really need to know the epistemic classes and the finer epistemic categories given here? How relevant are these for advancing their conceptual and procedural understanding, problem-solving ability, etc.? Would the latter not improve simply by going deeper into the subject and practicing more challenging problems rather than reflecting about these epistemic distinctions? Data reported here suggest that the more meritorious students (i.e., those who do well in standard tests) usually, though not always, do have this discriminating ability like experts do. But this is a correlation, not necessarily a causation. Is it of any significance?

To respond to these questions, let us first note an important point. In this work, by epistemic knowledge, we do not mean deeper subject-specific conceptual knowledge and competence. An example will clarify. Consider the principle of conservation of linear momentum of an isolated (multiparticle) system. As long as the student is aware that it is a physical law (P5) not a mathematical result and also distinct from other categories, we are fine. We do not care for our purpose here if one knows the deeper warrants, namely, that in Newtonian mechanics, it is a consequence of the second and third laws or, more generally, it follows from translational invariance, which in turn follows from the homogeneity of space, and so on. That for us amounts to having deeper subject-specific conceptual knowledge and higher-level technical competence. However, if Newton's laws are the starting inputs in a derivation, and suitable intermediate mathematical steps are given to lead to the principle of momentum conservation, the student should realize that the latter is a mathematical deduction (M2) of the laws (P5). Thus the epistemic dimension is being viewed as separate from the dimension of subject-specific conceptual depth and related technical competence. (Of course, these different dimensions are not completely orthogonal; at a very deep level they would be intertwined, but at the undergraduate stage, we may view the epistemic dimension as quite separate from the others.) But then why teach along that dimension at all?
The conjecture that epistemic distinctions are educationally relevant is based on some overlapping intuitions. First, epistemic discrimination is one (among many others) signature of expert-novice difference in physics. There is much emphasis currently in science education discourse on making implicit expert knowledge and practices explicit to students. This is generally thought to facilitate student transition to expertise. Second, the conceptual and the epistemic, though being viewed here along quite different dimensions from the point of view of the discipline, form a holistic web of student cognition. Thus it is plausible, though not certain, that discriminating ability along one dimension (epistemic) might affect the same kind of ability along the other (conceptual), i.e., may help conceptual discrimination that is necessary for proper learning of physics at any level. Third, insistence on precise words for distinct categories could generally reduce sloppiness in the way technical language is used in a physics classroom, and this again can be beneficial to students, especially to non-native speakers. But all this is a conjecture. Whether the conjecture is sensible and to what extent enhancing awareness of epistemic distinctions in precise words really helps can be seen only in a controlled pedagogic investigation in the future.

The pedagogic recommendation of this work then is that teachers highlight these categories in clear terms, as they work out the steps of standard derivations, solve problems, or explain other qualitative and quantitative arguments in physics, as much as feasible, given the time constraints. A formal teaching of these categories is, of course, not envisaged; they need to be appreciated in the context of particular derivations or problems. The precise wording of these categories, and indeed the coding scheme itself may vary to some extent from one teacher to another. What is important is to emphasize their discrimination and also debate the possible ambiguities that might be encountered occasionally.

One important advantage of the tool is that given the fixed template of epistemic categories, it should be relatively easy for any instructor to design an epistemic questionnaire of the kind illustrated here, in different topics. This is in contrast to the development of a concept inventory which usually needs considerable research inputs on student conceptual pitfalls in the topic to design sensible alternatives to the correct option in a multiple choice test. The suggested epistemic pedagogy is not hard to implement; it needs no new infrastructure and can be a part of any college teacher orientation program. Our preliminary trials with college teachers not reported here suggest that teachers find the exercise of assigning epistemic categories interesting. Whether and to what extent this would be so generally is to be seen in the future. Also, we envisage this to be only one simple reform among the many other important collateral reforms needed in undergraduate physics education. 
Another practical use of this kind of tool is in regard to the large scale public examinations in the country, where it can serve as a complementary tool to the usual conceptbased tests, or possibly as an initial or final screening test. The minor inherent ambiguities likely in an epistemic test could be conceivably taken care of in a suitable grading scheme or by interviews.

\section{Refinement of the tool}

The epistemic categories used in this work subsume those in our earlier work. Do they represent a maximal set of distinct epistemic categories? Looking through numerous derivations at college or university level physics, they seem to be so. The major divisions, nominal, physical, and mathematical, are natural and basic to the classification. But, undoubtedly there is always a scope for a slightly different way of subdividing them into further categories and phrasing them. In this connection, we should caution against confusing our epistemic categories with the different reasoning types (such as thought experiment, analogical reasoning, and model-based reasoning) sometimes invoked by cognitive historians of science [17]. The fundamental distinction to note is that our categories refer to individual propositions, not to any overall reasoning patterns or strategies. For example, analogical reasoning belongs here to the category of heuristic argument, while thought experiment and model-based reasoning will involve a number of different epistemic categories in our framework.

Thus apart from correcting some avoidable deficiencies of the present tool noted at the end of Sec. III, any substantial improvement of the tool lies mainly in enhancing the contexts of the derivations. However, we cannot just put together several different derivations in a single test-it will simply not be viable in terms of the time and student diligence required to complete the test. The only way out seems to be to depart from the practice of choosing one long derivation for the tool, but instead choose appropriate "chunks" of connected sequences of propositions from a judiciously chosen sample of derivations from a variety of topics spanning undergraduate physics. As a final effort in this series of investigations, a tool of this kind has been prepared for large scale administration, and the results will be reported in due course.

As a concluding remark, we note that modern scholarship in science education advocates a holistic approach to investigating student cognition which is viewed as a web of interconnections of concepts, epistemic and ontological beliefs, etc. [18]. Viewed from this perspective, a reductive approach of breaking down a complex argument in physics into individual propositions and likewise reducing the rich epistemic belief system of a student into distinct categories may seem naive. Indeed the issues surrounding the construct validity of our tool seem in part to arise from this reductive approach. A more holistic and qualitative approach of ascertaining content-based epistemic ability using student narratives and interviews might measure what we are looking for better. Yet we advocate simple tools of this kind or their refinements in teaching and assessment. This can be a first step to orient students to implicit expert epistemology in physics and should be easily implemented even in large scale educational systems that cannot afford expensive and elaborate infrastructure based reforms in physics education.

\section{ACKNOWLEDGMENTS}

We thank all the students who participated in this study, the teachers at different institutions who helped us administer the tool to their students, and the experts who participated in the validation exercise. It is a pleasure to thank the reviewers for their incisive comments on the earlier versions of this work, which spurred us to revise the original tool, carry out another independent survey, and clarify the various issues in the paper. We acknowledge the support of the Government of India, Department of Atomic Energy, under NIUS project of HBCSE-TIFR (No. 12-R\&D-TFR-6.04-0600). 


\title{
APPENDIX: DIAGNOSTIC TOOL FOR EPISTEMIC UNDERSTANDING
}

Tool 2, consisting of the orientation note, followed by the test is given in Figs. 14-18. Tool 1 was the same test, without the orientation note.

\section{Orientation Note \\ (Please read this note carefully.)}

\begin{abstract}
You must have frequently seen mathematical derivations in physics. A derivation consists of a sequence of steps. Here we call each step a proposition. We have three broad classes of propositions: Mathematical, Physical and Nominal, each split in a number of categories, as explained below.

In the accompanying test, a certain derivation is given in full. All you need to do is to assign each proposition in the given derivation to one of the following categories. For your convenience, the list of categories, each stated briefly, appears on every page of the test.

\begin{tabular}{|l|l|}
\hline \multicolumn{2}{|c|}{ Mathematical } \\
\hline M1 & $\begin{array}{l}\left.\text { A proposition may simply quote a mathematical formula or result (e.g. } \int \sec ^{2} x d x=\tan x\right) \text { fa- } \\
\text { miliar to anyone who knows the relevant mathematics but may not know the physical situation. } \\
\text { We call this a context-independent mathematical proposition. }\end{array}$ \\
\hline M2 & $\begin{array}{l}\text { A proposition may follow logically or mathematically from the earlier propositions (which } \\
\text { could be of any type: mathematical, physical or nominal). Using them, a person knowing } \\
\text { the relevant mathematics only should be able to deduce the given proposition. We call it a } \\
\text { mathematical/logical deduction with no further physical input. }\end{array}$ \\
\hline
\end{tabular}
\end{abstract}

\section{Physical}

A physical proposition, as the term suggests, has some physical input which may be of different kinds.

P1 It could be a mathematical definition of a physical quantity in terms of other physical quantities, e.g. definition of Young's modulus $Y=\frac{F / A}{l / L}$, where the meanings of symbols are given before, at the start or in the earlier propositions of the derivation.

P2 It may involve choice of a system, a frame of reference, directions of co-ordinate axes, etc., usually for convenience, which does not imply any assumptions.

P3 It may involve reasoning based on a diagram (not a mere reference to the diagram), which will be hard to replace by pure algebraic reasoning without using any diagram.

P4 It may involve assumption(s)/approximation(s)/idealization(s) regarding the system, its initial condition(s), etc., (in text or figure), that usually simplifies the situation (e.g. ignoring air resistance in motion, or using ideal gas law for a real gas).

P5 It may quote an experimentally or observationally established law/principle/relation or its deductions (e.g. Coulomb's law, the elliptical shape of planetary orbits) or measured values of quantities (say specific heat of a gas at a certain temperature, etc.)

P6 It may be just an educated guess of a physical result, a generalization that is not proved in the derivation but may seem plausible based on intuition, experience, etc. This is sometimes called 'heuristic reasoning'.

\begin{tabular}{|l|l|}
\hline \multicolumn{2}{|c|}{ Nominal } \\
\hline $\begin{array}{l}\text { A nominal proposition involves an arbitrary choice of words and symbols. Here 'arbitrary choice' } \\
\text { means a free choice not governed by any mathematical or physical reasoning. }\end{array}$ \\
\hline N1 & $\begin{array}{l}\text { This involves denoting physical quantites, or their combinations, by algebraic symbols (usually } \\
\text { from English or Greek alphabet), e.g. } \vec{L} \text { for angular momentum vector, } \eta \text { for viscosity. }\end{array}$ \\
\hline N2 & $\begin{array}{l}\text { This involves naming in words of a property of a system, a process, a relation, a condition, etc. } \\
\text { (e.g. calling a process adiabatic if it does not involve exchange of heat with the surroundings). }\end{array}$ \\
\hline N3 & $\begin{array}{l}\text { This involves adopting some arbitrary convention in the subject usually (though not necessarily) } \\
\text { shared in the physics community (e.g. taking the direction of current in a wire to be opposite } \\
\text { to that of the flow of electrons). }\end{array}$ \\
\hline
\end{tabular}

FIG. 14. Diagnostic tool for epistemic understanding: (I) Orientation note. Next parts of the tool in Figs. 15-18. 


\section{Test on Content-Epistemic Relation in Physics}

Instructions for the test:

- The test contains the derivation of a well-known relation in physics in terms of a series of propositions. A list of different types of propositions is provided separately as well as at the bottom of each page of the derivation. These types are grouped into three broad classes: Nominal (N1 to N3), Physical (P1 to P6), and Mathematical (M1 to M2).

- Against each proposition in the derivation, tick the most relevant (only one) proposition type from the given list (N1 to M2). In case of M2 being the proposition type, mention the serial number(s) of relevant preceding proposition(s) in brackets.

- Class/Year: Stream: Institution:

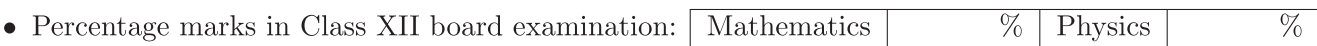

Types of propositions:

\begin{tabular}{|l|l|l|}
\hline \multirow{4}{*}{ Nominal } & N1 & $\begin{array}{l}\text { Denoting physical quantities / combination of physical quantities by algebraic sym- } \\
\text { bols. }\end{array}$ \\
\cline { 2 - 4 } & N2 & Naming in words of a property of a system / process / relation / condition. \\
\cline { 2 - 4 } & N3 & Arbitrary convention adopted in the subject. \\
\hline
\end{tabular}

\begin{tabular}{|c|c|c|}
\hline \multirow{6}{*}{ Physical } & $\mathbf{P} 1$ & $\begin{array}{l}\text { Mathematical / Quantitative definition of a physical quantity in terms of other } \\
\text { physical quantities. }\end{array}$ \\
\hline & $\mathbf{P 2}$ & Choice of system / axes / frame of reference, etc. \\
\hline & P3 & Diagram based reasoning / argument. \\
\hline & $\mathbf{P} 4$ & $\left.\begin{array}{l}\text { Idealisation } \\
\text { OR } \\
\text { Assumption(s) / Approximation(s) } \\
\text { OR } \\
\text { Simplified given condition(s) }\end{array}\right\}$ in text or figure. \\
\hline & P5 & $\begin{array}{l}\text { Experimentally known fact / relation / principle / law applicable in the situation } \\
\text { and deductions based on it. }\end{array}$ \\
\hline & P6 & $\begin{array}{l}\text { Unproved or heuristic argument in the given derivation. (Heuristic means not } \\
\text { strictly logical, but plausible.) }\end{array}$ \\
\hline \multirow{2}{*}{ Mathematical } & M1 & General formula or relation in mathematics, independent of the present context. \\
\hline & M2 & $\begin{array}{l}\text { Logical or mathematical deduction from one or more previous propositions without } \\
\text { further physical input. }\end{array}$ \\
\hline
\end{tabular}

FIG. 15. Diagnostic tool for epistemic understanding: (II) Instructions for the test and types of propositions. Note: (i) The actual test contained a few additional advisory notes that are omitted here for the sake of brevity. (ii) To keep it linguistically simple for the student, the phrase "proposition type" was used, which we refer to as "epistemic category" in this paper. (iii) For ease of reference, the list of proposition types was provided at the bottom of each page of the derivation, which is suppressed here. Next parts of the tool in Figs. 16-18. 


\section{Derivation of Bernoulli's Equation}

General notations used in the derivation:

(1) $t=$ Time

(2) $g=$ Acceleration due to gravity

(3) $m=$ Mass

(4) $\rho=$ Density

(5) $u=$ Flow speed

(6) $p=$ Pressure

(7) $\mathbf{F}=$ Force

(8) $d \mathbf{s}=$ Displacement

(9) $K=$ Kinetic energy

(10) $W=$ Work done by external force

(11) $\theta=$ Angle between $\mathbf{F}$ and $d \mathbf{s}$
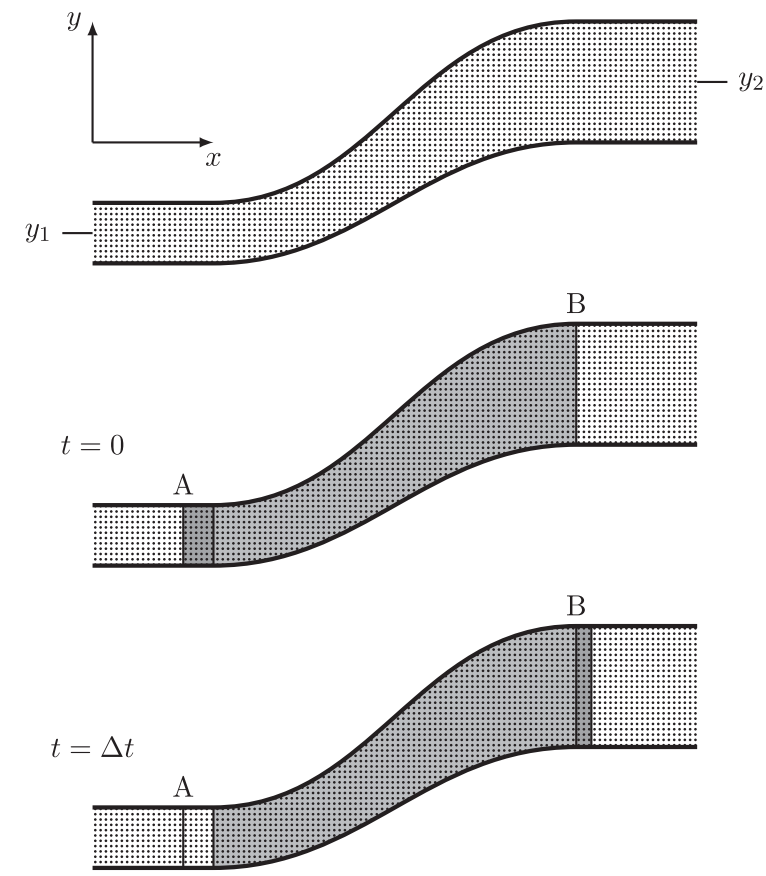

Consider the flow of a fluid in a tube of non-uniform cross section and varying elevation as shown in the figure.

(contd.)

FIG. 16. Diagnostic tool for epistemic understanding: (III) Derivation of Bernoulli's equation (part 1). Next parts of the tool in Figs. 17 and 18 . 


\section{Derivation of Bernoulli's Equation (contd.)}

\begin{tabular}{|c|c|c|}
\hline No. & Proposition & $\begin{array}{l}\text { Proposition } \\
\text { type }\end{array}$ \\
\hline S1 & $\begin{array}{l}\text { A fluid flow is said to be steady when its velocity at every point is constant in } \\
\text { time and normal to the cross section at that point. }\end{array}$ & $\mathrm{N} 2$ \\
\hline $\mathrm{S} 2$ & We consider the situation when the fluid flow is steady. & $\mathrm{P} 4$ \\
\hline S3 & The parts of the tube near ends A and B are horizontal. & $\mathrm{P} 4$ \\
\hline $\mathrm{S} 4$ & $\begin{array}{l}\text { We take the coordinate axes such that the } x-\text { axis is along the horizontal } \\
\text { direction and } y \text {-axis is along the vertically upward direction as shown in the } \\
\text { figure. }\end{array}$ & $\mathrm{P} 2$ \\
\hline S5 & $\begin{array}{l}u_{1}=\text { horizontal speed of the fluid at end } \mathrm{A} \\
u_{2}=\text { horizontal speed of the fluid at end B } \\
y_{1}=\text { elevation of fluid at end A } \\
y_{2}=\text { elevation of the fluid at end B } \\
p_{1}=\text { pressure on the fluid at end A } \\
p_{2}=\text { pressure on the fluid at end B }\end{array}$ & N1 \\
\hline S6 & $\begin{array}{l}\text { We consider the system to be the portion of the fluid between ends } \mathrm{A} \text { and } \mathrm{B} \\
\text { at the initial instant } t=0 \text {. }\end{array}$ & $\mathrm{P} 2$ \\
\hline S7 & Let $\Delta t$ be the arbitrarily small time interval after the initial instant $t=0$. & N1 \\
\hline S8 & $\begin{array}{l}\text { Let } \Delta V_{1} \text { be the volume of the fluid system that leaves end } \mathrm{A} \text { in the flow } \\
\text { direction (horizontal) and let } \Delta V_{2} \text { be the volume of the fluid system that } \\
\text { leaves end } \mathrm{B} \text { (in flow direction) in the same time } \Delta t \text {. }\end{array}$ & N1 \\
\hline S9 & Let $\rho_{1}$ be the density at $\mathrm{A}$ and $\rho_{2}$ at $\mathrm{B}$. & N1 \\
\hline S10 & $\begin{array}{l}\text { A fluid whose density remains the same independent of the pressure is called } \\
\text { an incompressible fluid. }\end{array}$ & $\mathrm{N} 2$ \\
\hline S11 & The fluid is taken to be incompressible. & $\mathrm{P} 4$ \\
\hline S12 & $\rho_{1}=\rho_{2}$ & M2 \\
\hline S13 & Let $\rho$ stand for the constant density of the fluid. & N1 \\
\hline S14 & $\begin{array}{l}\text { Since mass is conserved, the mass of the fluid system leaving end } \mathrm{A} \text { and the } \\
\text { mass leaving end } \mathrm{B} \text { in time } \Delta t \text { are equal. }\end{array}$ & $\mathrm{P} 5$ \\
\hline S15 & $\rho=\frac{\text { Mass }}{\text { Volume }}$ & $\mathrm{P} 1$ \\
\hline S16 & $\Delta V_{1}=\Delta V_{2}$ & M2 \\
\hline
\end{tabular}

(contd.)

FIG. 17. Diagnostic tool for epistemic understanding: (IV) Derivation of Bernoulli's equation (part 2). Note: Expert answers have been filled in this table. Next part of the tool in Fig. 18. 


\section{Derivation of Bernoulli's Equation (contd.)}

\begin{tabular}{|c|c|c|}
\hline No. & Proposition & $\begin{array}{l}\text { Proposition } \\
\text { type }\end{array}$ \\
\hline S17 & Let $\Delta V$ be the constant value of the above volumes. & $\mathrm{N} 1$ \\
\hline S18 & $K=\frac{1}{2} m u^{2}$ & $\mathrm{P} 1$ \\
\hline S19 & Let $\Delta K$ be the change in the kinetic energy of the fluid system in time $\Delta t$ & N1 \\
\hline S20 & $\begin{array}{l}\text { From figure, clearly } \Delta K \text { equals the difference in kinetic energy of the darker } \\
\text { shaded portions. }\end{array}$ & P3 \\
\hline $\mathrm{S} 21$ & $\Delta K=\frac{1}{2} \rho \Delta V\left(u_{2}^{2}-u_{1}^{2}\right)$ & M2 \\
\hline $\mathrm{S} 22$ & $W=\int_{\text {initial }}^{\text {final }} \mathbf{F} \cdot d \mathbf{s}$ & $\mathrm{P} 1$ \\
\hline $\mathrm{S} 23$ & $\mathbf{F} \cdot d \mathbf{s}=F d s \cos \theta$ & M1 \\
\hline S24 & Force due to gravity $=m g$ in the vertically downward direction. & P5 \\
\hline $\mathrm{S} 25$ & $g$ is taken to be a constant. & $\mathrm{P} 4$ \\
\hline $\mathrm{S} 26$ & In time $\Delta t$ work done by gravitational force $=-\rho \Delta V g\left(y_{2}-y_{1}\right)$ & M2 \\
\hline S27 & Pressure $=\frac{\text { Normal Force }}{\text { Area }}$ & $\mathrm{P} 1$ \\
\hline S28 & $\begin{array}{l}\text { A fluid is said to be non-viscous when the work done on flowing fluid is solely } \\
\text { due to pressure forces and gravity. }\end{array}$ & $\mathrm{N} 2$ \\
\hline S29 & We assume the fluid to be non-viscous. & $\mathrm{P} 4$ \\
\hline S30 & $\begin{array}{l}\text { In time } \Delta t \text { work done on the fluid due to pressure at end } \mathrm{A} \text { is } p_{1} \Delta V \text { and that } \\
\text { at end } \mathrm{B} \text { is }-p_{2} \Delta V\end{array}$ & M2 \\
\hline S31 & Total work done in time $\Delta t=\left(p_{1}-p_{2}\right) \Delta V-\rho \Delta V g\left(y_{2}-y_{1}\right)$ & M2 \\
\hline S32 & $\begin{array}{l}\text { According to Work-Energy theorem, total work done on a non-viscous fluid is } \\
\text { equal to the change in its kinetic energy. }\end{array}$ & P5 \\
\hline S33 & $\frac{1}{2} \rho \Delta V\left(u_{2}^{2}-u_{1}^{2}\right)=\left(p_{1}-p_{2}\right) \Delta V-\rho \Delta V g\left(y_{2}-y_{1}\right)$ & M2 \\
\hline S34 & $\frac{1}{2} \rho u_{1}^{2}+p_{1}+\rho g y_{1}=\frac{1}{2} \rho u_{2}^{2}+p_{2}+\rho g y_{2}$ & M2 \\
\hline S35 & The derivation can be generalised for any two points in the fluid system. & $\mathrm{P} 6$ \\
\hline S36 & $\frac{1}{2} \rho u^{2}+p+\rho g y=$ constant at every point in the fluid. & M2 \\
\hline
\end{tabular}

FIG. 18. Diagnostic tool for epistemic understanding: (V) Derivation of Bernoulli's equation (part 3). Note: Expert answers have been filled in this table. 
[1] C. Stathopoulou and S. Vosniadou, Exploring the relationship between physics-related epistemological beliefs and physics understanding, Contemp. Educ. Psychol. 32, 255 (2007).

[2] F. C. Mercan, Epistemological beliefs of physics undergraduate and graduate students and faculty in the context of a well-structured and ill-structured problem, Ph.D. thesis, Ohio State University, 2007.

[3] D. Hammer, Epistemological beliefs in introductory physics, Cognit. Instr. 12, 151 (1994).

[4] D. Hammer, Two approaches to learning physics, Phys. Teach. 27, 664 (1989).

[5] E. F. Redish, Teaching Physics with the Physics Suite (Wiley, New York, 2003).

[6] E. F. Redish, R. N. Steinberg, and J. M. Saul, Student expectations in introductory physics, Am. J. Phys. 66, 212 (1998).

[7] W. K. Adams, K. K. Perkins, N.S. Podolefsky et al., New instrument for measuring student beliefs about physics and learning physics: The Colorado learning attitudes about science survey, Phys. Rev. Phys. Educ. Res. 2, 010101 (2006).

[8] A. Sirnoorkar, A. Mazumdar, and A. Kumar, Students' epistemic understanding of mathematical derivations in physics, Eur. J. Phys. 38, 015703 (2017).

[9] S.E. Toulmin, The Uses of Argument (Cambridge University Press, Cambridge, England, 1958).

[10] T. J. Bing, An epistemic framing analysis of upper-level physics students' use of mathematics, Ph.D. thesis, University of Maryland, 2008, https://www.physics.umd.edu/ perg/dissertations/Bing/.
[11] K. J. Knoespel, Models and diagrams within the cognitive field, in Model Based Reasoning in Scientific Discovery, edited by L. Magnani, N. J. Nersessian, and P. Thagard (Kluwer Academic/Plenum Publishers, New York, 1999).

[12] D. L. Schwartz and J. Heiser, Spatial representations and imagery in learning, in The Cambridge Handbook of the Learning Sciences, edited by R. K. Sawyer (Cambridge University Press, Cambridge, England, 2006).

[13] L. Cohen, L. Manion, and K. Morrison, Research Methods in Education (Routledge, New York, 2011).

[14] See Supplemental Material at http://link.aps.org/ supplemental/10.1103/PhysRevPhysEducRes.16.010103 for distribution of responses to each of the 36 individual propositions, for the whole sample as well as for the three groups, GH, GM, and GL.

[15] National Curriculum Framework-2005, National Focus Group Position Paper on Examination Reform (National Council of Educational Research and Training, New Delhi, 2006).

[16] Report of the Committee on Evaluation Reforms (University Grants Commission, India, 2018).

[17] N. J. Nersessian, in Model Based Reasoning in Scientific Discovery, edited by L. Magnani, N. J. Nersessian, and P. Thagard (Kluwer Academic/Plenum Publishers, New York, 1999).

[18] T. G. Amin, C. L. Smith, and M. Wiser, Student conceptions and conceptual change, in Handbook of Research in Science Education, edited by N. G. Lederman and S. K. Abell (Routledge, London, 2014), Vol. II. 